\title{
Subject-variability effects on micron particle deposition in human nasal cavities
}

\author{
H. Calmet ${ }^{\mathrm{a}, *}$, C. Kleinstreuer ${ }^{\mathrm{b}}$, G. Houzeaux ${ }^{\mathrm{a}}$, A.V. Kolanjiyil ${ }^{\mathrm{b}}$, O. Lehmkuhl ${ }^{\mathrm{a}}$, \\ E. Olivares ${ }^{a}$, M. Vázquez ${ }^{a}$ \\ a Barcelona Supercomputing Center (BSC-CNS), Department of Computer Applications in Science and Engineering, Edificio NEXUS I, Gran Capitán 2- \\ 4, 08034 Barcelona, Spain \\ b Department of Mechanical and Aerospace Engineering, North Carolina State University, Raleigh, 27695 NC, United States
}

\section{A R T I C L E I N F O}

\section{Keywords:}

Nasal cavities

Particle transport/deposition

Computational fluid-particle dynamics

\begin{abstract}
A B S T R A C T
Validated computer simulations of the airflow and particle dynamics in human nasal cavities are important for local, segmental and total deposition predictions of both inhaled toxic and therapeutic particles. Considering three, quite different subject-specific nasal airway configurations, micron-particle transport and deposition for low-to-medium flow rates have been analyzed. of special interest was the olfactory region from which deposited drugs could readily migrate to the central nervous system for effective treatment. A secondary objective was the development of a new dimensionless group with which total particle deposition efficiency curves are very similar for all airway models, i.e., greatly reducing the impact of intersubject variability. Assuming dilute particle suspensions with inhalation flow rates ranging from 7.5 to $20 \mathrm{~L} / \mathrm{min}$, the airflow and particle-trajectory equations were solved in parallel with the in-house, multi-purpose Alya program at the Barcelona Supercomputing Center. The geometrically complex nasal airways generated intriguing airflow fields where the three subject models exhibit among them both similar as well as diverse flow structures and wall shear stress distributions, all related to the coupled particle transport and deposition. Nevertheless, with the new Stokes-Reynolds-number group, $S t k^{1.23} R e^{1.28}$, the total deposition-efficiency curves for all three subjects and flow rates almost collapsed to a single function. However, local particle deposition efficiencies differed significantly for the three subjects when using particle diameters $d_{p}=2,10$, and $20 \mu \mathrm{m}$. Only one of the three subject-specific olfactory regions received, at relatively high values of the inertial parameter $d_{p}^{2} Q$, some inhaled microspheres. Clearly, for drug delivery to the brain via the olfactory region, a new method of directional inhalation of nanoparticles would have to be implemented.
\end{abstract}

\section{Introduction}

The pulmonary route for direct drug-aerosol delivery is an attractive approach to combat brain or lung diseases or to reach systemic regions. Of great potential is optimal targeting of solid tumors or severely inflamed areas with multifunctional particles promising lower side-effects and costs than other treatment options, such as chemotherapy or radiation (Kleinstreuer, Feng, \& Childress, 2014; Kolanjiyil \& Kleinstreuer, 2016; Kolanjiyil, Kleinstreuer, \& Sadikot, 2016). For example, intranasal direct drug delivery is being considered as a possible and effective route to deliver vaccines, insulin, and medication for treating various

\footnotetext{
* Corresponding author.

E-mail address: hadrien.calmet@bsc.es (H. Calmet).
} 
diseases and disorders affecting the central nervous system (Illum, 2000; Illum, Tsuda, \& Gehr, 2015; Mistry, Stolnik, \& Illum, 2009). In case of intranasal drug delivery, it is quite challenging for the inhaled drugs to reach the olfactory region with the possibility of crossing the blood-brain barrier in order to reduce/eliminate brain tumors or to maximize the impact on the central nervous system (Dhuria, Hanson, \& Frey, 2010; Thorne, Emory, Ala, \& Frey, 1995). Even though intranasal delivery provides greater advantages, the drug-amount actually reaching the brain is quite low, but possibly higher than intravenous administration (Garcia, Schroeter, \& Kimbell, 2015; Schroeter, Kimbell, \& Asgharian, 2006; Thorne et al., 1995; Thorne, Pronk, Padmanabhan, \& Frey, 2004). Clearly, intranasal targeted drug delivery to a specific location could improve delivery efficiency (Inthavong, Tian, Tu, Yang, \& Xue, 2008; Shi, 2007; Shi, Kleinstreuer, \& Zhang, 2008, 2007). In general, high particle deposition at any predetermined site depends largely on the given airway geometry as well as the fluid-particle inlet conditions, including the breathing mode and the type of inhaler employed (Keeler, Patki, Woodard, \& Frank-Ito, 2016; Schroeter et al., 2006; Segal, Kepler, \& Kimbell, 2008). The complex geometrical structure of the nasal cavity makes it difficult to predict the airflow and aerosol transport (Schroeter, Garcia, \& Kimbell, 2010). Additionally, the geometrical variability among individuals raises significant challenges in developing efficient drug delivery devices (Garcia, Tewksbury, Wong, \& Kimbell, 2009; Inthavong et al., 2008; Kimbell et al., 2007; Leong, Chen, Lee, \& Wang, 2010).

Deposition of inhaled aerosols in human nasal cavity has been extensively studied using in vivo (Bennett \& Zeman, 2005; K.-H. Cheng et al., 1996; Y. S. Cheng et al., 1996; Cheng, Yeh, \& Swift, 1991; Kesavan, Bascom, Laube, \& Swift, 2000; Kesavanathan \& Swift, 1998; Kesavanathan, Bascom, Laube, \& Swift, 1998; Rasmussen, Andersen, \& Pedersen, 2000; Wiesmiller et al., 2003) in vitro (Cheng et al., 2001; Cheng, 2003; Cheng, Cheng, Yeh, \& Swift, 1995; Garcia, Tewksbury, Wong, \& Kimbell, 2009; L. Golshahi, M. L. Noga, R. B. Thompson, \& W. H. Finlay, 2011; Kelly, Asgharian, Kimbell, \& Wong, 2004a, 2004b; Schroeter, Tewksbury, Wong, \& Kimbell, 2015a; Storey-Bishoff, Noga, \& Finlay, 2008; Zwartz \& Guilmette, 2001) and in silico (Corley et al., 2015; Dastan, Abouali, \& Ahmadi, 2014; Garcia et al., 2015; Inthavong et al., 2006; Inthavong, Wen, Tian, \& Tu, 2008; Kimbell et al., 2007; Liu, Matida, \& Johnson, 2010; Schroeter et al., 2006, 2010; Schroeter, Garcia, \& Kimbell, 2011; Shanley, Zamankhan, Ahmadi, Hopke, \& Cheng, 2008; Shi et al., 2008; Shi, 2007; Shi, Kleinstreuer, \& Zhang, 2007; Wang, Hopke, Ahmadi, Cheng, \& Baron, 2008; Xi \& Longest, 2008; Xi, Kim, Si, Corley, \& Zhou, 2016; Zhang \& Kleinstreuer, 2011) methods. The deposition results from these studies indicate that there exist significant variations in human nasal aerosol deposition. While the major reason for these variations is due to anatomical variations, differences in experimental techniques can also affect reported nasal aerosol deposition outcome (Kelly et al., 2004a, 2004b; Schroeter, Garcia, \& Kimbell, 2011; Shi et al., 2007). Even though an in vivo deposition measurement on human subjects is the most physiologically realistic method, there are many limitations. Such experiments are restricted due to the usage of aerosols which may lead to side effects, especially when using radioactive aerosols, and hence are limited in the number of trials. Additionally, in vivo measurements cannot clearly provide detailed regional deposition measurements and the presence of subject variability limits comparative analyses without geometrical correlations (Y. S. Cheng et al., 1996; Rasmussen et al., 2000). Even though these limitations can be overcome with in vitro experiments, even small differences in the in vitro model geometry can significantly alter aerosol deposition. Recent investigations have shown that surface irregularities (surface roughness vs. surface smoothness) due to the differences in the fabrication process and/or due to the low resolution of the scanned images have resulted in significant variations in aerosol deposition (Dastan et al., 2014; Kelly et al., 2004a, 2004b; Schroeter et al., 2011; Shi et al., 2007). Hence, as an alternative to these experimental techniques numerical analysis, using Computational Fluid-Particle Dynamics (CF-PD), has shown many advantages including repeatability and regional deposition resolution (Kolanjiyil \& Kleinstreuer, 2016, 2013). Recent developments in Magnetic Resonance Imaging (MRI) and Computer Tomography (CT) techniques have helped in reconstructing physiologically realistic models. Such subject-specific geometries can be coupled with the advancements in CF-PD simulation computer hardware and software technology to obtain detailed, accurate and realistic visualization of the flow field and particle transport/deposition (Kleinstreuer et al., 2014). For example, for direct drug delivery numerical analysis concerning nasal airway models has been able to reveal detailed nasal airflow fields (Garcia, Bailie, Martins, \& Kimbell, 2007; Kim, Na, Kim, \& Chung, 2013; Kimbell, Frank, Laud, Garcia, \& Rhee, 2013), particle dynamics (Dastan et al., 2014; Garcia et al., 2015; Inthavong et al., 2006, 2008; Kimbell et al., 2007; Liu et al., 2010; Schroeter et al., 2006; Shanley et al., 2008; Shi et al., 2007, 2008; Shi, 2007; Wang et al., 2008; Xi \& Longest, 2008; Xi et al., 2016; Zhang \& Kleinstreuer, 2011), dosimetry of inhaled vapours (Asgharian, Price, Schroeter, Kimbell, \& Singal, 2012; Morris, HAssETT, \& Blanchard, 1993; Schroeter et al., 2008), and odourant delivery (Keyhani, Scherer, \& Mozell, 1997). It can also assist in nasal surgery (Garcia et al., 2007; Kimbell et al., 2013; Rhee, Pawar, Garcia, \& Kimbell, 2011), intranasal drug delivery and development of nasal drug delivery devices (Inthavong et al., 2006; Inthavong, Tian, Tu, Yang, \& Xue, 2008b; Keeler et al., 2016; Kimbell et al., 2007).

As indicated, the results from nasal deposition studies have shown that the nasal aerosol deposition is a function of inhalation conditions and particle properties, including size, shape and density (Schroeter, Tewksbury, Wong, \& Kimbell, 2015b; Shi et al., 2007). These studies suggest that the nasal passage acts as a filtering mechanism for the incoming particles which leads to large deposition in the anterior part, thereby reducing drug aerosols from reaching their predetermined areas (Garcia \& Tewksbury, 2009; Liu et al., 2010). In the inertial regime (for particle diameters $\geq 1 \mu \mathrm{m}$ ) the aerosol deposition increases with the particle size and air flow rate, following a sigmoidal curve with very low deposition for lower micron particles (Shi et al., 2007a). For nanoparticles $(1-100 \mathrm{~nm})$, aerosol deposition decreases with diameter due to higher diffusivity of smaller particles leading to higher nasal deposition (Garcia et al., 2015; Shi et al., 2008a). Even though nasal deposition has been investigated for different inhalation conditions and particle sizes, only a limited number of studies have focused on estimating the regional distribution of the deposited particles (Dastan et al., 2014; Garcia et al., 2015; Schroeter et al., 2006; Shanley, Zamankhan, Ahmadi, Hopke, \& Cheng, 2008; Shi et al., 2007a; Zwartz \& Guilmette, 2001) and it is still unknown how the regional distribution depends on subject variability. Subject variability in nasal deposition hinders development of intranasal therapeutic drugs, because variability in deposition leads to variability in drug dose and its reaction (Garcia, Tewksbury, et al., 2009). 
As a first step towards the overall goal of optimal intranasal drug delivery, ultimately to the central nervous system, this paper focuses on the effects of subject-variability on both airflow and regional drug deposition, considering microspheres in three very different nasal cavities. A secondary objective is to develop representative particle deposition graphs which are common to the majority of human nasal cavities. Many investigations have been conducted to map the anatomical differences among individuals (Guilmette, Cheng, \& Griffith, 1997). Other studies, using methods like acoustic $\backslash$ optical rhinometry and rhinomanometry as well as MRI and CT techniques, have shown large variations in nasal cross-sectional area and nasal resistance (Corey, Gungor, Liu, Nelson, \& Fredberg, 1998; Corey, Gungor, Nelson, Fredberg, \& Lai, 1997; Menache et al., 1997). Preliminary results from in vivo and in vitro methods have confirmed the individual differences in nasal anatomy which leads to intersubject variability in aerosol deposition (Bennett \& Zeman, 2005; K.-H. Cheng et al., 1996; Cheng et al., 1991, 1995; Y. S. Cheng et al., 1996; Cheng, 2003; Garcia, Tewksbury, et al., 2009; Kesavan et al., 2000; Kesavanathan \& Swift, 1998; Kesavanathan, Bascom et al., 1998; Rasmussen et al., 2000; Wiesmiller et al., 2003). Recently, investigations using CF-PD modeling methodology also predicted subject variability in nasal deposition (Dastan et al., 2014; Garcia, Schroeter, et al., 2009; Keeler et al., 2016; Wang et al., 2008). A number of attempts have been made to define functional correlations for nasal deposition with geometrical parameters like nostril shape and dimension, minimum cross-sectional area, and \or transnasal pressure drop (Cheng et al., 1991; Y. S. Cheng et al., 1996; Garcia, Tewksbury, et al., 2009; Golshahi et al., 2011; Kesavan et al., 2000; Kesavanathan \& Swift, 1998). Even though these correlations did not completely eliminate the scatter in inter-individual nasal deposition, some have been able to reduce the variations (Garcia, Tewksbury, et al., 2009; Golshahi et al., 2011). However, these studies were based on a limited number of samples and hence these functional forms should be tested with more cases. While investigations considering subject variability have been conducted to predict the total nasal deposition, variations in regional deposition have not been made available. The objective of the present investigation is to determine the impact of intersubject variability in spatial and regional deposition distributions of micron particles in three different human nasal cavities.

\section{Theory}

\subsection{Nasal geometries}

In order to evaluate the effect of subject variability on regional particle deposition distribution, three nasal airway geometries have been considered. The first nasal model (subject A) was developed from MRI scans of the nose of a healthy, 53-year-old, nonsmoking male (weighing $73 \mathrm{~kg}, 173 \mathrm{~cm}$ tall), provided by CIIT (Research Triangle Park, NC; also refer to Kelly et al. (2004a), Shi et al. (2008, 2007), Shi (2007). The image file was then processed and surface-smoothed to develop a CAD-like geometry file (Shi et al., 2007a). The second model (subject B) was developed by Kabilan et al. (2016). Subject B model was developed from multi-slice CT scans of the nose of a healthy, 35-year-old male weighing $68 \mathrm{~kg}$. and $173 \mathrm{~cm}$ tall. The details regarding image segmentation and geometry development are provided in Kabilan et al. (2016). The third model (subject C) was developed by Liu, Johnson, Matida, Kherani, and Marsan (2009). This standardized geometry of the human nasal cavity was created by computing and averaging 30 sets of computed tomography (CT) scans of nasal airways of healthy subjects. All the details of the nasal cavity standardized are provided in Liu et al. (2009).

Table 1 summerizes the geometrical features and dimensions for the three subjects. Length, height, area, volume and their ratios help to represent the $3 \mathrm{D}$ objects and also to compare them. While subject A and B are balanced with $\mathrm{L} / \mathrm{H}$ ratio almost equal to 2 , subject $\mathrm{C}$ has the most stretch nasal cavity of the three patients and the lowest $\mathrm{A} / \mathrm{V}$ ratio.

\subsection{Mesh generation}

The mesh discretisation used for scientific computing and simulation of the passages of the nasal cavity must be of high quality. An unstructured mesh was employed to represent faithfully the complex shape of the computational domain. Specifically using ANSYS ICEM CFD (ANSYS Inc., USA) an octree-based method was used to generate a high resolution surface mesh. Surface smoothing was performed using successive Laplace smoothing (Field, 1988) and aspect ratio (ratio of shortest to longest edge) smoothing (ANSYS Meshing User's Guide, 2000) in order to avoid surface shrinkage Fig. 1.

The second step of the meshing process was the generation of a volumetric mesh, for which the Delaunay method was employed. This yielded a tetrahedral volume mesh with a smooth cell transition ratio $(\sim 1.2)$ close to the boundary wall. Finally a multi-layer prism mesh was created to resolve the high velocity gradients at the wall, with a few pyramids needed to ensure the transition between the prism layer and tetrahedral mesh in the core of the computational domain. (see Table 2).

\section{Table 1}

Geometrical features of the nasal cavities, with $\mathrm{L}=$ length $(\mathrm{cm}), \mathrm{H}=$ height $(\mathrm{cm}), \mathrm{A}=$ area $\left(\mathrm{cm}^{2}\right)$ and $\mathrm{V}=$ volume $\left(\mathrm{cm}^{3}\right)$ then geometrical features of nostrils, with $\mathrm{NL}=$ length $(\mathrm{cm}), \mathrm{NW}=$ width $(\mathrm{cm})$ and $\mathrm{P}=$ perimeter $(\mathrm{cm})$ of the nostril.

\begin{tabular}{|c|c|c|c|c|c|c|c|c|c|c|}
\hline Subject & Structural features & $\mathrm{L}$ & $\mathrm{H}$ & $\mathrm{L} / \mathrm{H}$ & A & $\mathrm{V}$ & $\mathrm{A} / \mathrm{V}$ & NL & NW & $\mathrm{P}$ \\
\hline A & Smoothness surface, slightly converging left nasal cavity & 9.5 & 4.1 & 2.3 & 430 & 18.8 & 22.8 & 1.2 & 0.4 & 3 \\
\hline B & Roughness surface, slight septum deviation & 10.6 & 4.8 & 2.2 & 538 & 26.2 & 20.5 & 1.7 & 0.4 & 3.8 \\
\hline $\mathrm{C}$ & symmetric standardized geometry & 10.9 & 3.8 & 2.8 & 188 & 29 & 6.5 & 1.7 & 0.6 & 3.9 \\
\hline
\end{tabular}




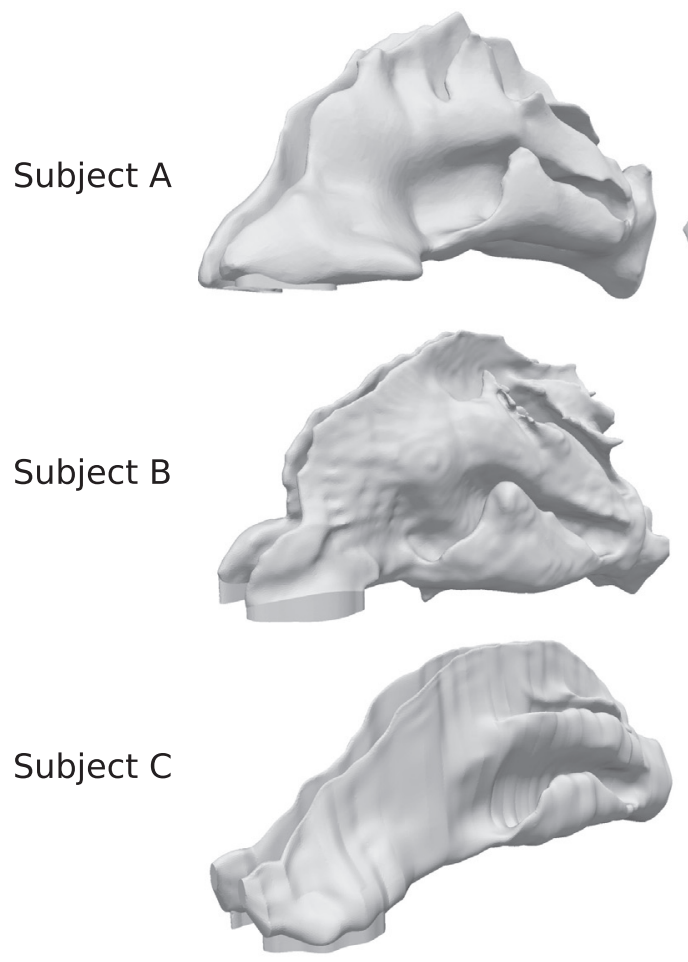

Right view
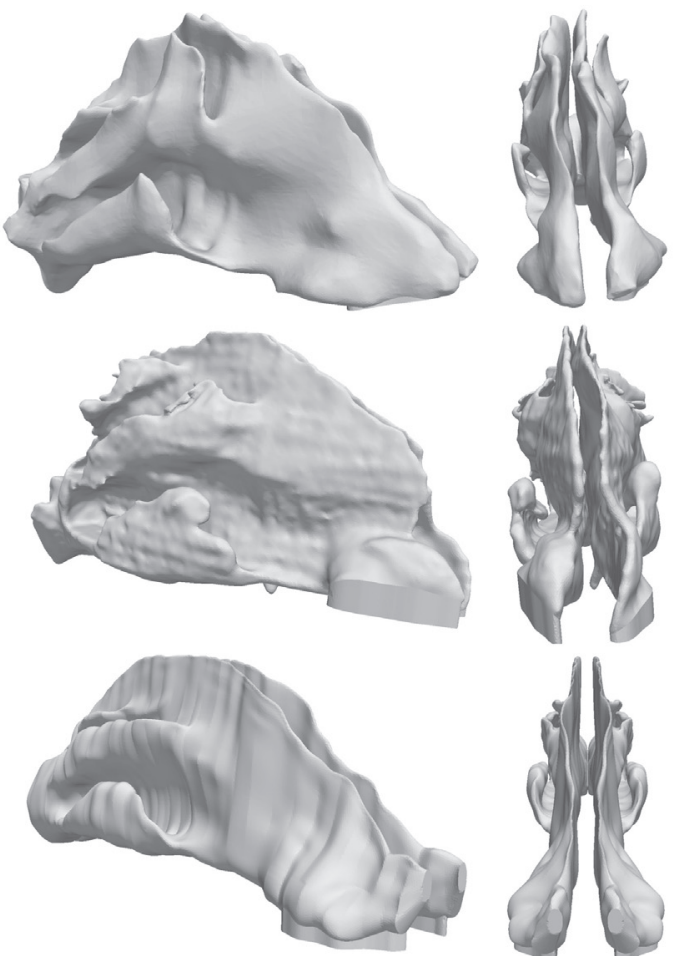

Left view

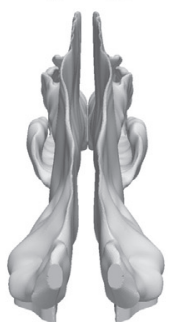

Front
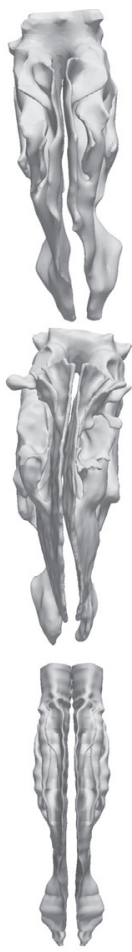

Top

Fig. 1. Geometry descriptions of the subjects.

\section{Table 2}

Summary of different mesh resolutions and simulation parameters with $N_{N}$ : number of nodes, $N_{E}$ : number of elements, $\Delta t$ : time step, $h$ : mean edge length of elements, $h_{p l 1}$ : height of the first element in the prism layer, $N_{p l}$ : number of prism layers and $h_{p l}$ : height of total prism layer.

\begin{tabular}{llllll}
\hline Mesh & $N_{N}\left(\times 10^{6}\right)$ & $N_{E}\left(\times 10^{6}\right)$ & $\Delta t(m s)$ & $h(m m)$ & $h_{p l}(\mu m)$ \\
\hline Subject A & 1.8 & 6.1 & 0.1 & 0.5 & 30 \\
Subject B & 2.0 & 6.4 & 0.1 & 0.5 & 30 \\
Subject C & 1.4 & 4.6 & 0.1 & 0.5 & 5 \\
\hline
\end{tabular}

The three different meshes are presented in Fig. 2. For all, a minimum element height of $30 \mu m$ at the wall for the most critical region (nasal valve). That corresponds to a range of value of the inner variable $y^{+}=\frac{u_{*} y}{v}$ of $1-3$ for a constant flow rate of $20 L / m i n$. These values of $y^{+}$can be considered to be sufficiently small for resolving the near-wall flow dynamics (Robinson, 1991) and falls into the range defined by Piomelli and Balaras (2002) that describes well resolved wall-layers. Mesh generation was accomplished with ANSYS ICEM CFD software requiring 2 GB of RAM on a single Intel Xeon X5650 core.

\subsection{Governing equation}

\subsubsection{Fluid solver}

In this section, we briefly describe the numerical method used to solve the Navier-Stokes equations with the Alya system (Alya System, 2000), which is a high-performance computational mechanics code developed at Barcelona Supercomputing Center (Houzeaux \& Vázquez, 2008). Assuming incompressible fluid flow, the task is to solve for the velocity vector $\boldsymbol{u}$ and pressure $p$ in a domain $\Omega$

$$
\begin{aligned}
& \rho \frac{\partial \boldsymbol{u}}{\partial t}+\rho(\boldsymbol{u} \cdot \nabla) \boldsymbol{u}-\nabla \cdot[2 \mu \varepsilon(\boldsymbol{u})]+\nabla p=\mathbf{0}, \\
& \nabla \cdot \boldsymbol{u}=0
\end{aligned}
$$

subject to initial and boundary conditions. Here $\mu$ is the viscosity of the fluid, and $\rho$ is the fluid density. The velocity strain rate is $\varepsilon(\boldsymbol{u})=\frac{1}{2}\left(\nabla \boldsymbol{u}+\nabla \boldsymbol{u}^{t}\right)$.

The numerical model to solve these equations is based on a stabilized finite element method. The stabilization is based on the 


\section{Subject A}

\section{Subject B}

\section{Subject C}
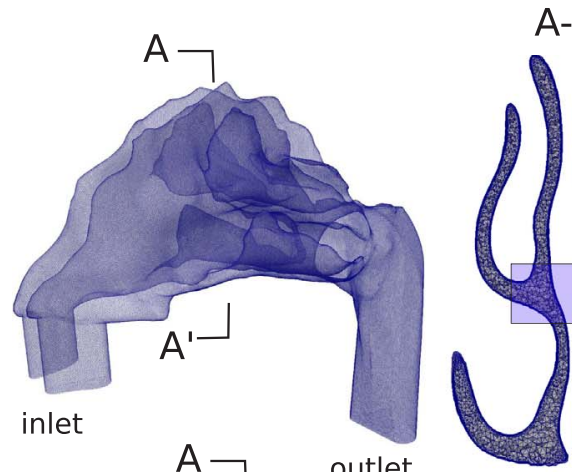

A-A'
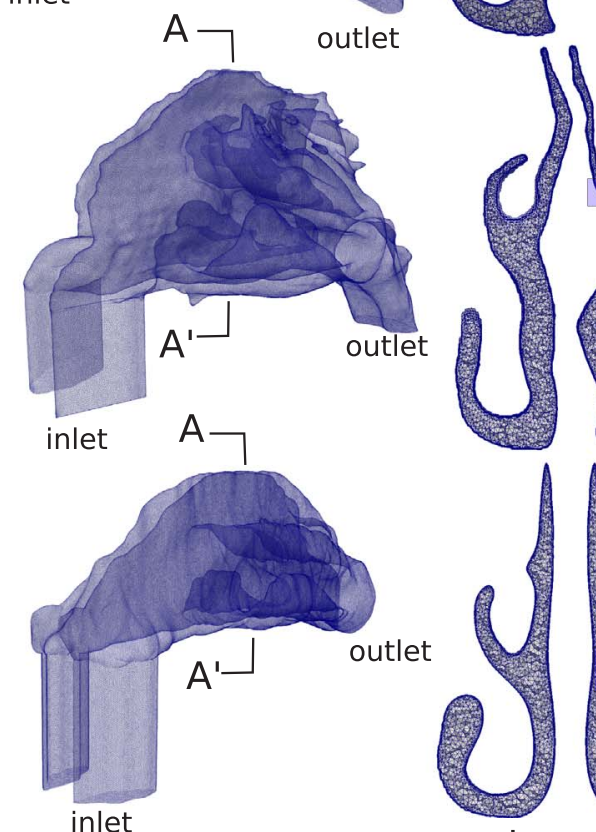

3D mesh

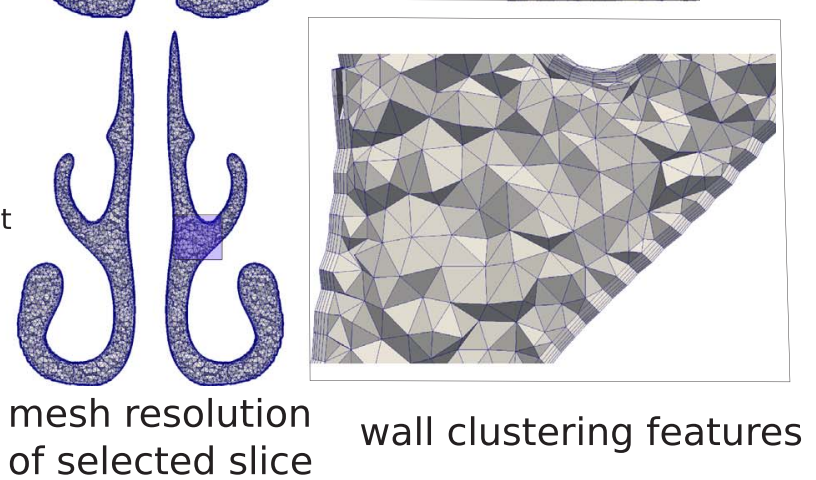

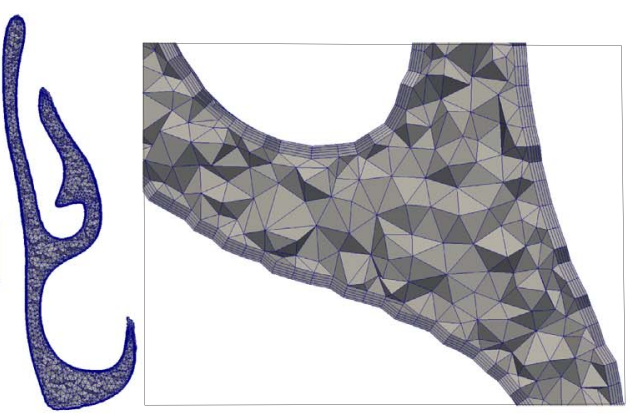
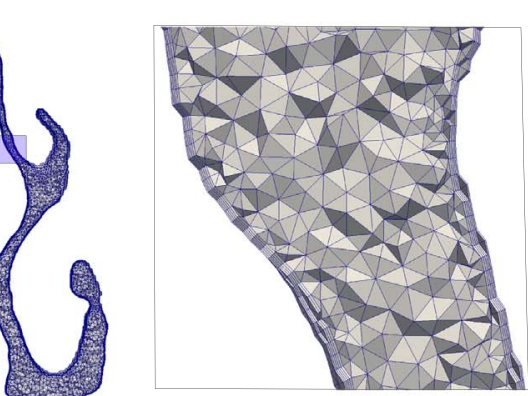

Fig. 2. Grid generation topology.

Variational MultiScale method which is considered as an implicit Large Eddy Simulation method (Colomés, Badia, Codina, \& Principe, 2015). The formulation is obtained by splitting the unknowns into grid scale and subgrid scale components. In the present formulation of Alya, the subgrid scale is, in addition, tracked in time and in space, thereby giving more accuracy and more stability to the numerical model (Houzeaux \& Principe, 2008).

Discretization of the Navier-Stokes equations yields a coupled algebraic system to be solved at each linearization step within a time loop. The resulting system is split to solve the momentum and continuity equations independently. This is achieved by applying an iterative strategy to solve for the Schur complement of the pressure. According to the present scheme, at each linearization step it is necessary to solve the momentum and continuity equation twice. This split strategy is described and validated in Houzeaux, Aubry, and Vázquez (2011).

The momentum equation algebraic system is solved using the GMRES method, with a simple diagonal preconditioner with a relatively low Krylov dimension. Usually, convergence is obtained within ten iterations. The continuity equations is solved using the Deflated Conjugate Gradient (DCG) method (Lohner, Mut, Cebral, Aubry, \& Houzeaux, 2011), together with a linelet preconditioner to accelerate the convergence in the boundary layers (Soto, Löhner, \& Camelli, 2003).

The simulation code is parallelized using a hybrid MPI + OpenMP approach. The partition of the mesh is carried out on-the-fly with the METIS library (Karypis, 2015). The fluid solver consists mainly of two basic steps, the assembly of the matrix representing the discretized form of the Navier-Stokes equations, and the solution of this system using the iterative solvers described previously. The assembly step does not require any communication, and its speedup is directly related to the load balance provided by METIS. The MPI exchanges in the iterative solvers are carried out in an asynchronous way, enabling to overlap work and communication during the matrix-vector products. The parallelization of the DCG solver is based on MPI gather functions, as extensively described in Lohner et al. (2011). Finally, parallel I/O is achieved using the HDF5 library (Folk, Cheng, \& Yates, 1999) in order to postprocess the velocities and pressure on the mesh. 


\subsubsection{Particle transport and deposition modeling}

Physical model The transport of particles is simulated in a Lagrangian frame of reference, following each particle individually. The main assumptions to develop the model are:

- Particles are sufficiently small and the suspension is dilute to neglect their effect on airflow: i.e., a one way coupling is appropriate;

- Particles are spherical and do not interact with each other;

- Particle rotation is negligible;

- Thermophoretic forces are negligible;

- The forces considered are drag $\boldsymbol{F}_{d}$, gravitational and buoyancy $\boldsymbol{F}_{g}$;

Let $\boldsymbol{x}_{p}, \boldsymbol{u}_{p}, \boldsymbol{a}_{p}$ be the particle position, velocity and acceleration, respectively. Let $m_{p}$ be its mass, $\rho_{p}$ its density, $d_{p}$ its diameter, and $V_{p}$ its volume. Particles are transported solving Newton's second law, and by applying the series of forces mentioned previously:

$$
\boldsymbol{a}_{p}=\left(\boldsymbol{F}_{d}+\boldsymbol{F}_{g}\right) / m_{p}
$$

The equation for the drag force assumes the particle has reached its terminal velocity and is given by

$$
\boldsymbol{F}_{d}=-\frac{\pi}{8} \mu d_{p} C_{d} \operatorname{Re}\left(\boldsymbol{u}_{p}-\boldsymbol{u}_{f}\right)
$$

where Re is the particle Reynolds number involving its relative velocity with the fluid:

$$
\operatorname{Re}=\frac{\left|\boldsymbol{u}_{p}-\boldsymbol{u}_{f}\right| d_{p}}{\nu}
$$

The drag coefficient is given by Ganser's formula Ganser (1993):

$$
\begin{aligned}
C_{d} & =\frac{24}{\operatorname{Re} k_{1}}\left(1+0.1118\left(\operatorname{Re} k_{1} k_{2}\right)^{0.6567}\right)+0.4305 \frac{k_{2}}{1+3305 /\left(\operatorname{Re} k_{1} k_{2}\right)}, \\
k_{1} & =\frac{3}{1+2 \psi^{-0.5}}, \\
k_{2} & =10^{1.84148\left(-\log _{10}(\psi)\right)^{0.5743}} \\
\psi & =\text { sphericity, }(=1 \text { for a sphere }) .
\end{aligned}
$$

The gravity and buoyancy forces contribute to the dynamic of the particle whenever there exist a density difference:

$$
\boldsymbol{F}_{g}=V_{p} \mathbf{g}\left(\rho_{p}-\rho\right)
$$

with $\mathbf{g}$ being the gravity vector.

Time integration Let us assume the particle path must be computed from time step $n$ to the next one $n+1$, where the time step size is defined as $\delta t:=t^{n+1}-t^{n}$.

The time integration to actualize the particle dynamics at the new time step $n+1$ is based on the Newmark method. This method is quite general in the sense that given the acceleration at time steps $n$ and $n+1$, one can obtain the velocity and position at the new time step $n+1$ in an explicit form, using $\boldsymbol{a}_{p}^{n}$, or implicit form using a linear combination between $\boldsymbol{a}^{n}$ and $\boldsymbol{a}_{p}^{n+1}$. The switch between these possibilities are two parameters, namely $\beta$ and $\gamma$ which thus enable to control the accuracy and stability of the method. Given the accelaration computed at time step $n+1$, the scheme consists in solving the following equations:

$$
\begin{aligned}
& \boldsymbol{a}_{p}^{n+1}=\boldsymbol{F}^{n+1} / m_{p}, \\
& \boldsymbol{u}_{p}^{n+1}=\boldsymbol{u}_{p}^{n}+\delta t\left(\gamma \boldsymbol{a}_{p}^{n+1}+(1-\gamma) \boldsymbol{a}_{p}^{n}\right), \\
& \boldsymbol{x}_{p}^{n+1}=\boldsymbol{x}_{p}^{n}+\boldsymbol{u}^{n} \delta t+\frac{1}{2} \delta t^{2}\left(2 \beta \boldsymbol{a}_{p}^{n+1}+(1-2 \beta) \boldsymbol{a}_{p}^{n}\right) .
\end{aligned}
$$

The drag force given by Eq. (4) depends on the relative velocity of the particle with respect to the fluid at $n+1$, and therefore to the position of the particle at the new time step. For robustness purpose, we apply a Newton-Raphson scheme to converge the nonlinearity in velocity, but not in position. Once the velocity is obtained, the position is updated.

In order to gain additional control on the particle path, an adaptive time step strategy is adopted. The strategy is based on an error estimation of the Newmark-scheme (Olivares \& Houzeaux, 2016).

Numerical implementation The particle transport computation can be carried out in both a single-code or multi-code version. In the latter case, particles are transported using the same code and MPI partition as the fluid; in the last case, two instances of the code are used to enable asynchornism. The parallelization is hybrid, based on MPI + OpenMP, together with a dynamic load balance mechanism, as particle are likely to be located in very few MPI processes. The parallel strategy is extensively described in Houzeaux et al. (2016).

Particle deposition The particle deposition efficiency is defined as: 


$$
\eta=\frac{N_{d e p}}{N_{\text {in }}}
$$

where $N_{d e p}$ is the number of particles deposited on the surface area of interest, which can be the total nasal passage wall or local surface areas, e.g., olfactory regions, and $N_{\text {in }}$ is the total number of particles released at the nostrils.

\subsubsection{Boundary and initial conditions}

For the inlet condition situated in the extended nostril, see Fig. 2, a constant velocity profile giving a steady flow rate of $20 \mathrm{~L} / \mathrm{min}$ was used. For the outlet, a zero pressure gage opening boundary condition was applied and finally a no-slip boundary condition were imposed on the all passage walls. The present study assumes constant air flow inhalation with equal flow rates assigned to both nostrils.

In order to inject particles at the accurate location and optimize the deposition, injectors were located at each nostril as a plane surface. Particles are distributed uniformly and the initial velocities of particles are assumed to be the same as the airflow velocity at the nostril. Furthermore, released particles at each time step would be a prohibitively expensive computationally approach. Instead, 'frozen' particle tracking was adopted here, i.e in the 'frozen' implicit LES computational domain, particles are released and fully tracked. Based on the literature (Ghalati et al., 2012; Shi, Kleinstreuer, \& Zhang, 2007b) and convergence test thousands of particles (7000) for each size are released to reach a constant deposition value independent of the number of injected particles.

\section{Model validations}

\subsection{Analysis of grid convergence}

The grid convergence study was performed with a dimensionless velocity magnitude profile at the location in a nasal valve slice indicated in Fig. 3, considering three different mesh resolutions of subject A, i.e., a coarse mesh (1 M; 1 million of elements), a medium mesh (6 M; 6 millions of elements) and a fine mesh (50 M; 50 millions of elements).

The fine mesh was produced using the Mesh Multiplication technique described in Houzeaux, de la Cruz, Owen, and Vázquez (2013). This technique consists in refining the mesh uniformly, recursively, on-the-fly and in parallel, inside the simulation code. For tetrahedra, hexahedra and prisms, each level multiplies the number of elements by eight, while a pyramid is divided into ten new elements. The finest mesh was obtained using a one-level mesh multiplication from the medium mesh (6 M), therefore obtaining approximately (due to the presence of pyramids) eight times more elements. The time to produce this multiplied mesh in parallel is almost negligible (Houzeaux et al., 2013).

The velocity profiles over an arbitrary line at a cross-section $16 \mathrm{~mm}$ distal from the nostril and for a constant inhalation flow rate of $20 \mathrm{~L} / \mathrm{min}$ are shown in Fig. 3 for different grid sizes. The profiles look very similar for the medium mesh and the fine mesh, although some discrepancies are still visible for the two higher grid sizes. The medium-size mesh with 6 million elements, strikes a balance between computational costs and accuracy of solution, and hence was used to provide the majority of results presented in this study.

\subsection{Particle deposition}

Particle deposition in the nasal cavity was compared to the experimental data reported by Kelly, Asgharian, Kimbell, and Wong (2004) as depicted in Fig. 4. The human nasal cast is based on the same MRI file as employed for the present study (i.e, the subject A) and the same impaction parameter (IP) was used, i.e.,

$$
I P=d_{p}^{2} \cdot Q
$$
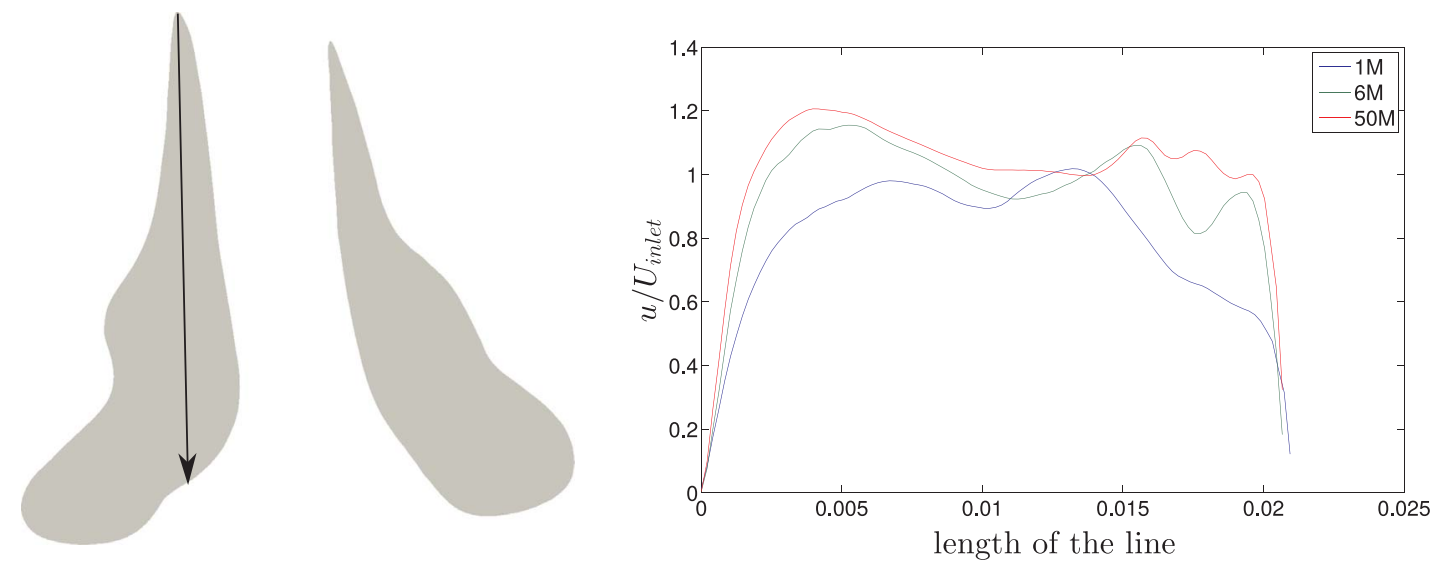

Fig. 3. Effect of computational grid size on calculated velocity profile for an arbitrary line in the flow $(Q=20 L / \mathrm{min})$. 


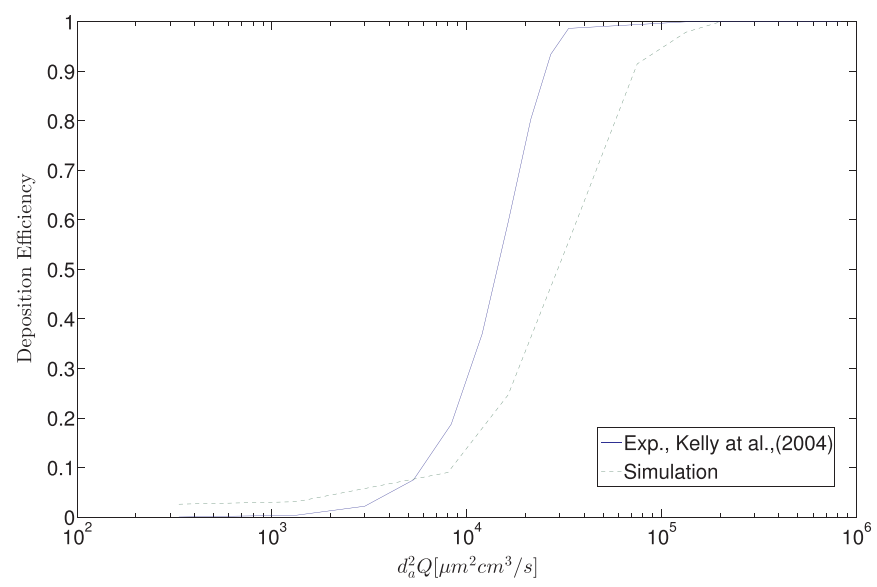

Fig. 4. Particle deposition efficiency comparison between simulation and experiment.

where $d_{a}$ is the particle aerodynamic diameter and $Q$ is the volumetric airflow rate. The aerodynamic diameter is that of a sphere with unit density $\left(1 \mathrm{~g} / \mathrm{cm}^{3}\right)$ and its mass is equal to the mass of the actual particle.

Fig. 4 shows an acceptable agreement between the present simulation and the measurements of Kelly et al. (2004). Differences in deposition results are due to the coarser airway surfaces in the replica producing higher deposition efficiencies than the numerical model (Shi et al., 2007b).

\section{Results and discussion}

\subsection{Airflow field results}

Assuming steady laminar flow with $Q_{i n}=20 \mathrm{~L} / \mathrm{min}$, the airflow fields for the three subjects are given in Figs. 5-7. It should be noted that the dimensionless velocities $u / U_{\text {inlet }}$ are the vector components normal the slice planes $1-1^{\prime}$ to $6-6^{\prime}$. Furthermore the location of slice planes for the three subjects are chosen to be approximately equivalent for direct comparison.

The airflow field description was already published in previous studies (Shi, Kleinstreuer, \& Zhang, 2008b, 2007b) for subject A at $Q=20 \mathrm{~L} / \mathrm{min}$. Thus, the analysis focuses on the comparison of the three subjects. Also the two first slices are displayed with the unity range of the dimensionless velocity and the rest of the slices are ranged zero to 0.75 . This configuration is done to reproduce the
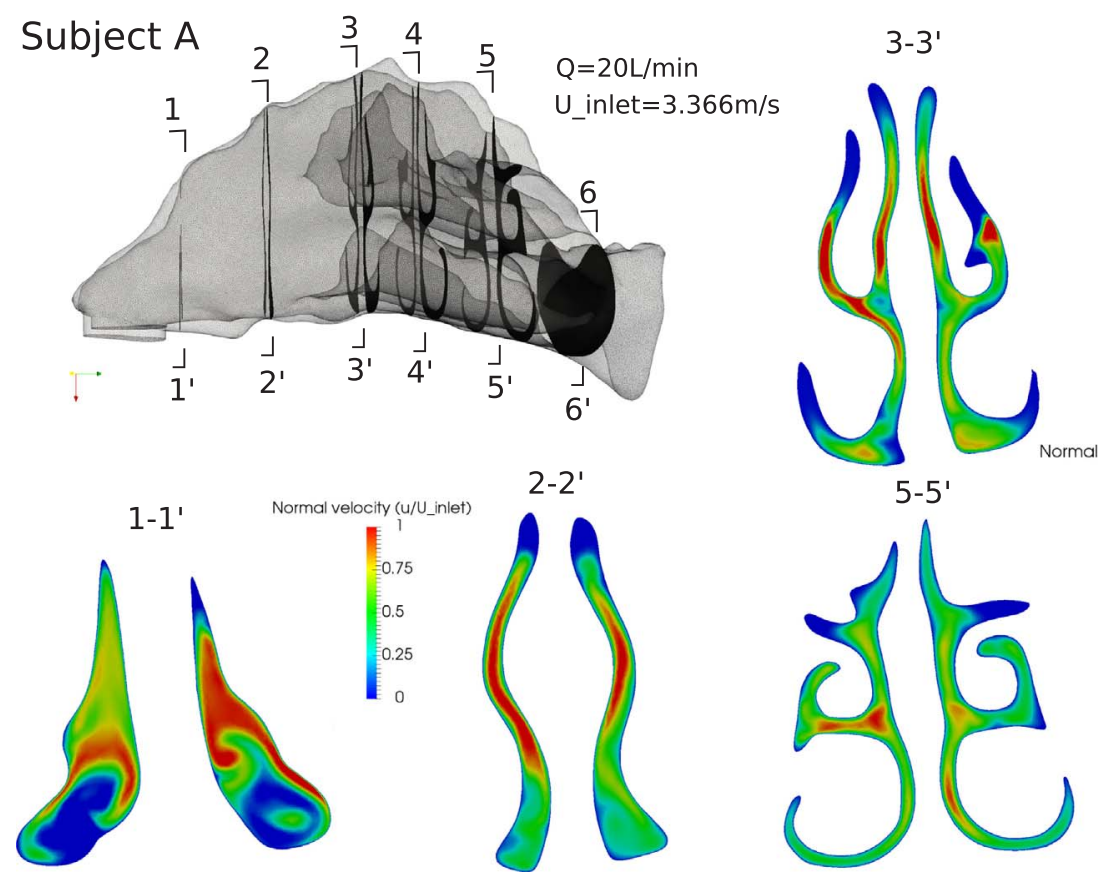

$6-6^{\prime}$

Fig. 5. Velocity fields for subject A at a constant inlet flow rate of $20 \mathrm{~L} / \mathrm{min}$ : velocity contours of velocity fieds in six selected slices. 

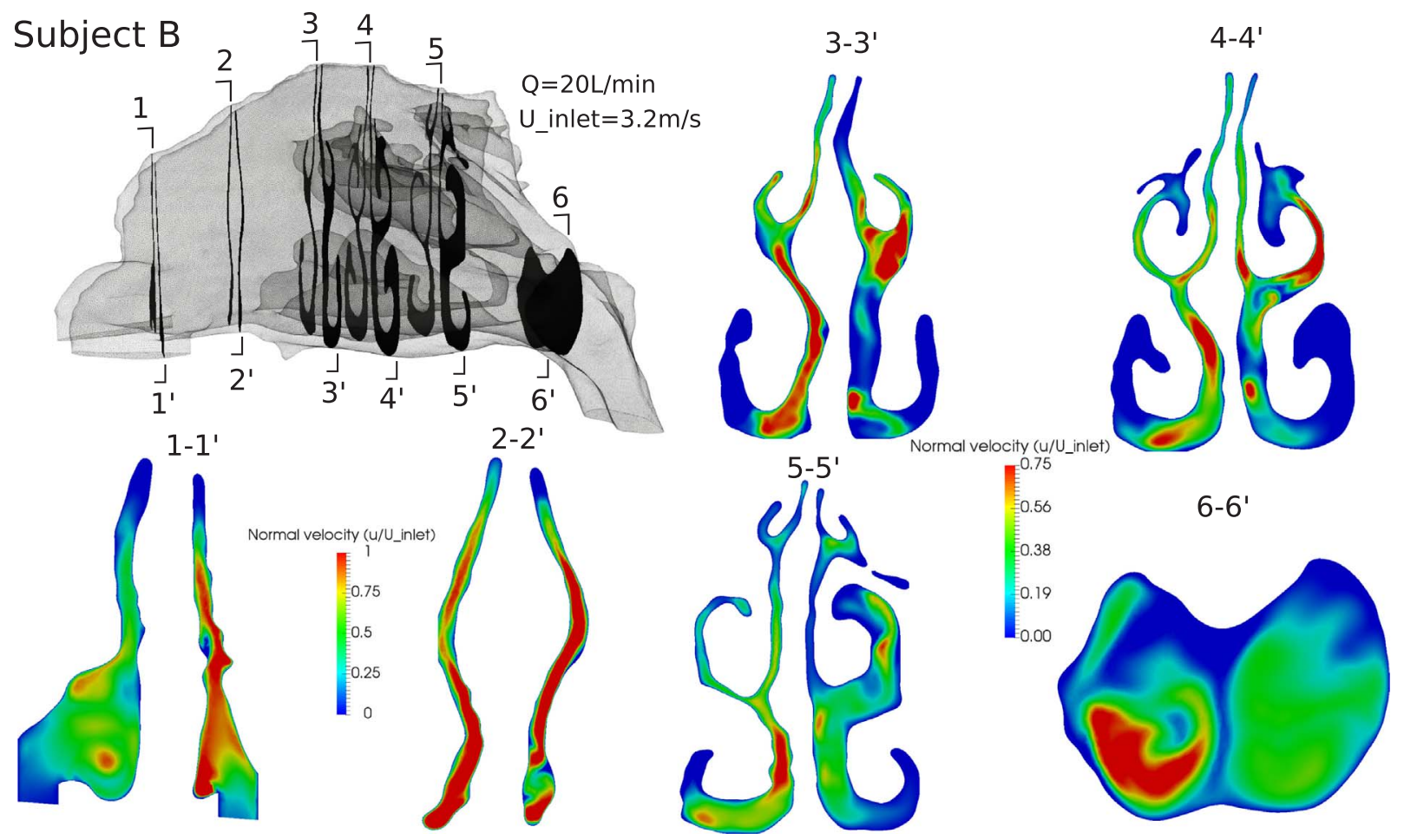

Fig. 6. Velocity fields for subject B at a constant inlet flow rate of $20 \mathrm{~L} / \mathrm{min}$ : velocity contours of velocity fieds in six selected slices.
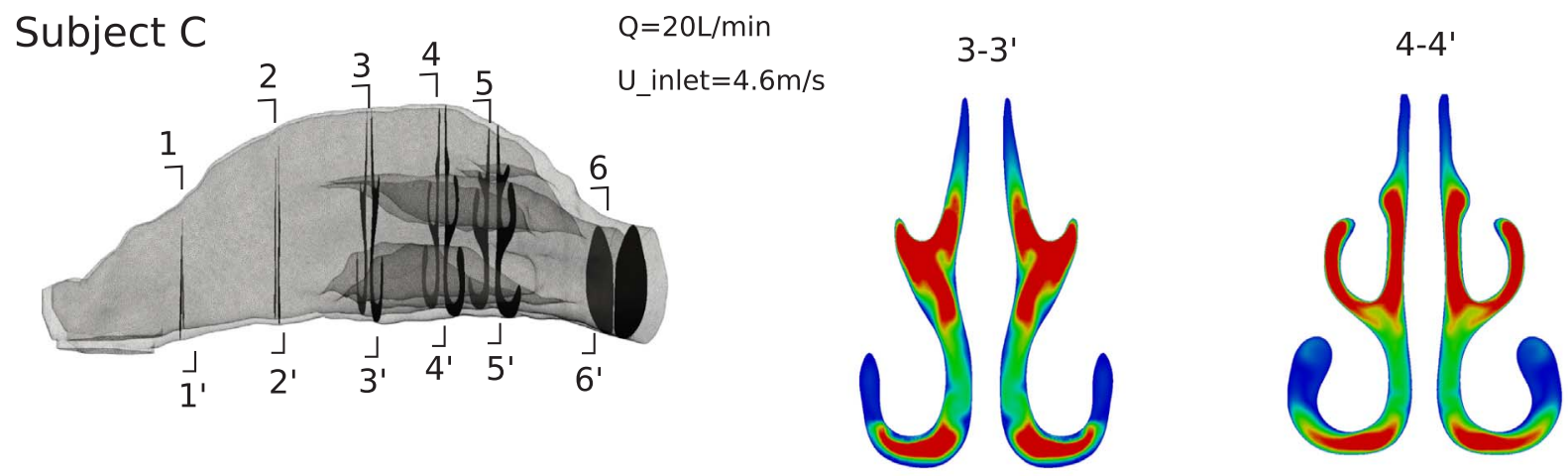$$
2-2^{\prime}
$$
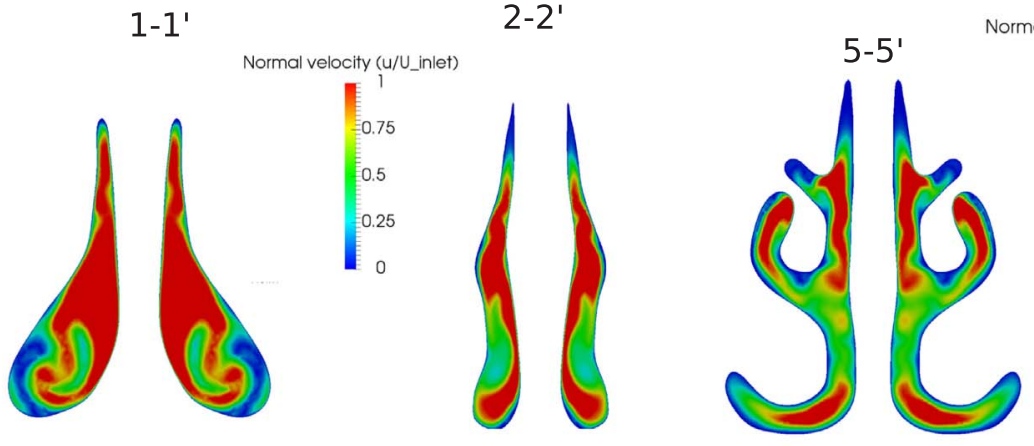

$6-6^{\prime}$

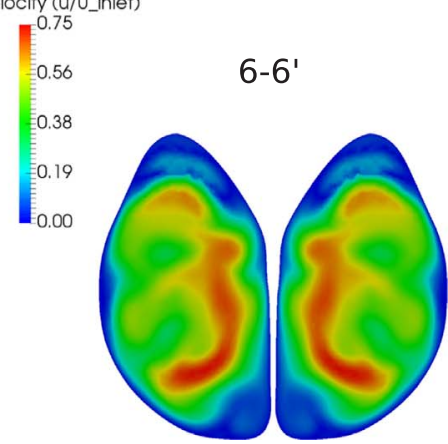

Fig. 7. Velocity fields for subject $\mathrm{C}$ at a constant inlet flow rate of $20 \mathrm{~L} / \mathrm{min}$ : velocity contours of velocity fieds in six selected slices.

layout of the cited previous studies.

Concerning slice 1-1', the tendency of higher velocity is observed in the left nasal cavity for the first two subjects, while for subject $\mathrm{C}$ velocity field is symmetric. By imposing the same inflow for each nostril, the differences between the left/right cross-sections 


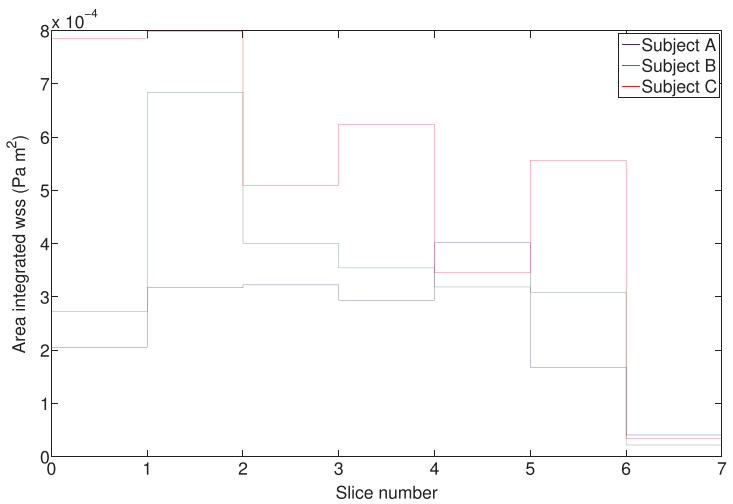

(a)

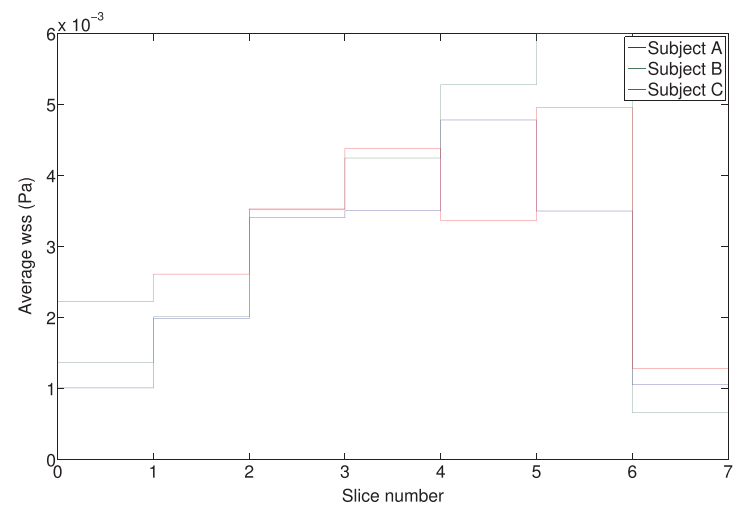

(b)
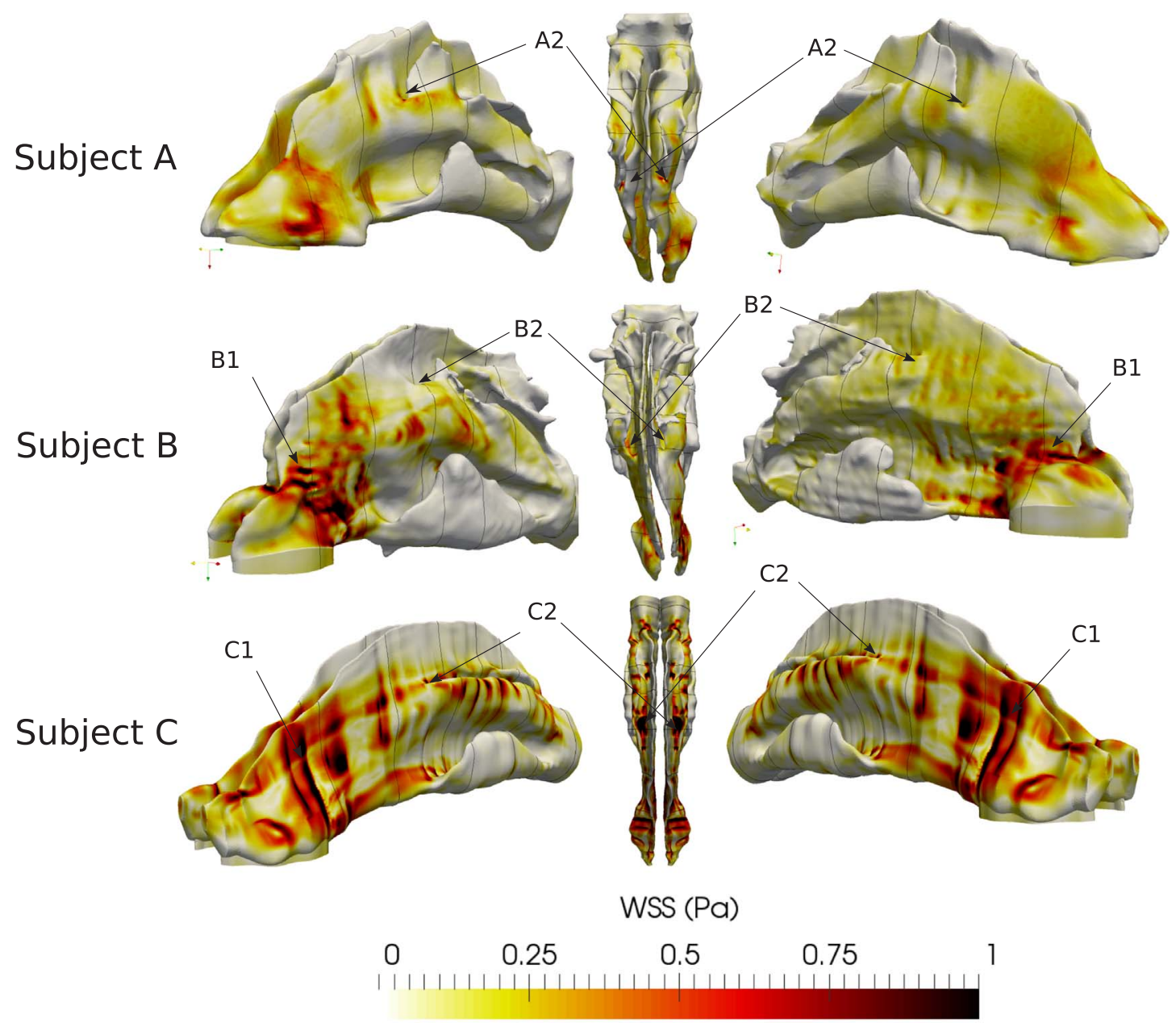

(c)

Fig. 8. (a) The surface integrated wall-shear stress value over slice sections. (b) Average wall-shear stress (i.e the surface integrated wall-shear stress divided by the considered area surface) value over slice sections. (c) Map of wall-shear stress with different views for the three subjects.

explains these elevated velocities.

These higher local flow rates are due to the small cross-sectional area of the nasal valve leading to a jet like flow (Calmet et al., 2016; Doorly, Taylor, Gambaruto, Schroter, \& Tolley, 2008). These narrow undulated and intricate passageways produce drastically 


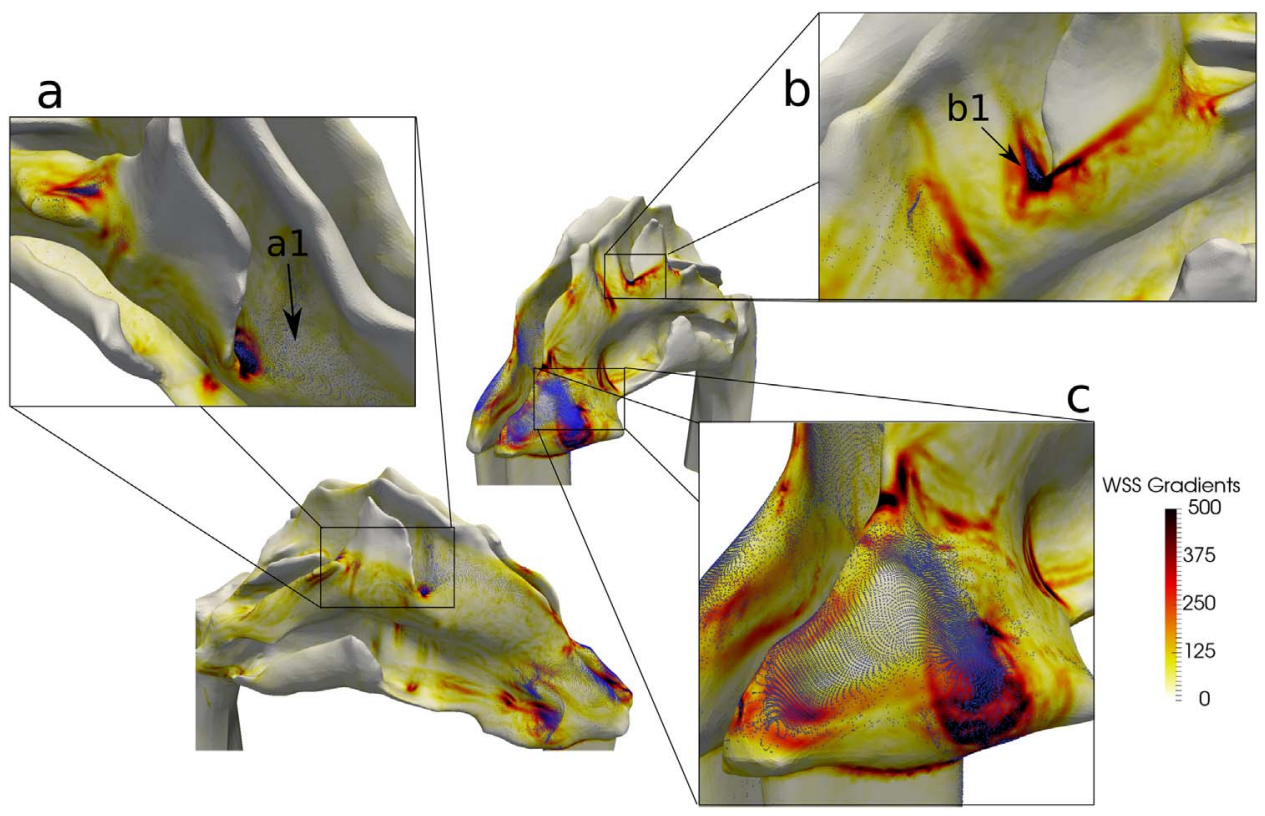

Fig. 9. Particle deposition pattern and map of wall-shear stress gradient for Subject A with details of interesting zone.

different flow fields. For example, for subject A and C, the nasal-valve jet is located on the superior part of the slice. The geometry of subject B is more narrow and undulated than the others, (see Fig. 6), causing a less established airflow where the nasal-valve jet is spread along the channel.

The bulk airflow for slice $2-2^{\prime}$ is located in the superior part for subject A. In contrast, the narrow channels of subject B expand the jet along all the passageways, as for the wider channels of subject $\mathrm{C}$.

The flow distributions for cross-Sections 3-3' to 5-5' are significantly different between the three subjects, covering the meatuses. Subject A exhibits high symmetric flow occurring in the superior part of the main passageway, decreasing progressively in the middle meatus while Subject B shows an asymmetric flow distribution, one part in the left middle meatus and the rest along the right center line of the main passageway. The rapid change of width of the channels and the septum deviation produce complex developing airflow. Subject $\mathrm{C}$ with the unusual geometric symmetry, has the bulk flow divided on two locations, i.e., middle part and inferior part of meatus and passageway.

In the cross-Section 6-6', where the two different flows of the left and right cavities join with different velocities begins the nasopharynx. Again, the flow distribution is significantly different for the three subjects. Subject A features asymmetric airflow and high flow rates in to the posterior part due to Dean vortices, resulting from the curved passage undergoing an almost $90^{\circ}$ bend from the horizontal nasal passage to the vertical descending sections. Two different and symmetric flows are observed for subject B with a clear separation in the middle.

In summary, the air flow entering in the nostrils change drastically when encountering the nasal-valve, i.e., almost from vertical to horizontal, where the diameter is reduced. This feature is observed with all subjects, although, the three geometries produce measurable differences. Then, most of the inhaled air flows through the wider middle-to-low portion of the main passageway which is free of obstacles. At the wall surface due to the no-slip condition, in conjunction with the actual widths of the meatuses and main passageway, interesting boundary-layer and near-wall flow establish. In the nasopharynx the left and right cavity-flows mix together before reaching the lungs.

\subsection{Wall-shear stress}

The wall-shear stress is a key parameter directly related to the resistive forces during respiration, the exchange processes and the near-wall flow field (Gambaruto, Doorly, \& Yamaguchi, 2010). Fig. 8 shows the wall-shear stress distributions of the three subjects with plots of the surface integrated wall-shear stress and the average wall-shear stress, i.e the surface integrated wall-shear stress divided by the considered area surface.

The highest wall-shear stress distribution and surface integrated wall-shear stress are observed for subject C. As expected, the largest flow resistance occurs between slices 1-2 (see Fig 8a) which corresponds to the nasal valve. The jet nasal valve, see Section 4.1, produces for subjects B and C some local maxima in skin friction on the thin and undulating channels downstream of the nasal valve, (see B1 and C1 of Fig 8c). This jet impinges on the anterior protrusion of the middle turbinate, creating a local maximum of wall-shear stress visible for all subjects (see A2, B2 and C2 in Fig 8c with the top view). The complex, undulating wall shapes of the turbinates in the mid-region of the nasal cavity affect the airflow by partitioning it into different regions. The undulation surfaces also favor exposing core flow to the nasal lining, enhancing exchange processes between lining and the flow as well as deposition of 


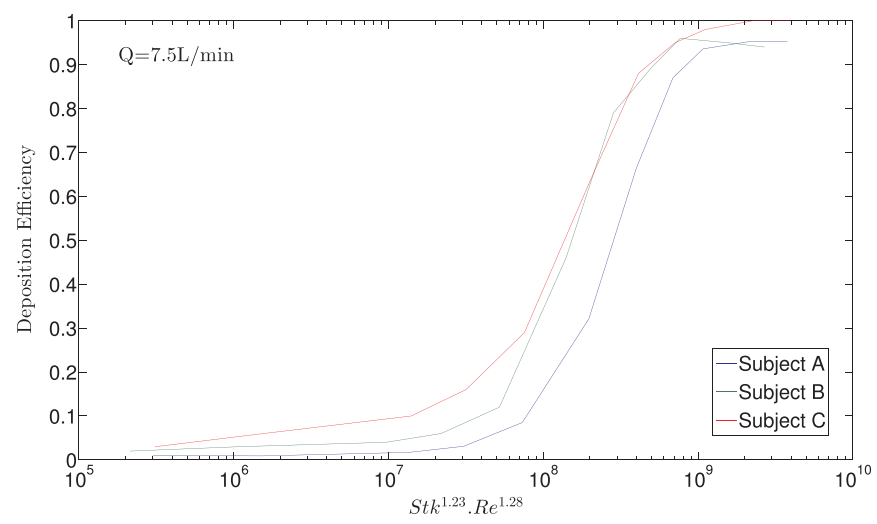

(a)

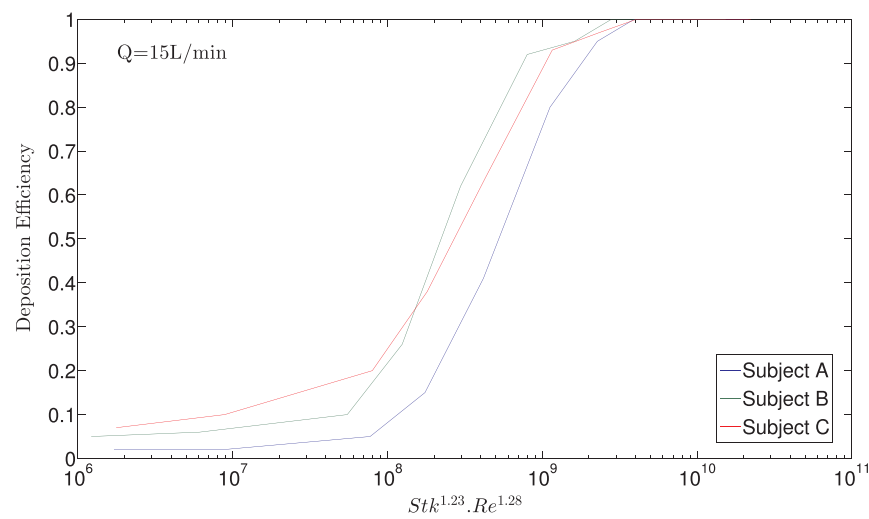

(b)

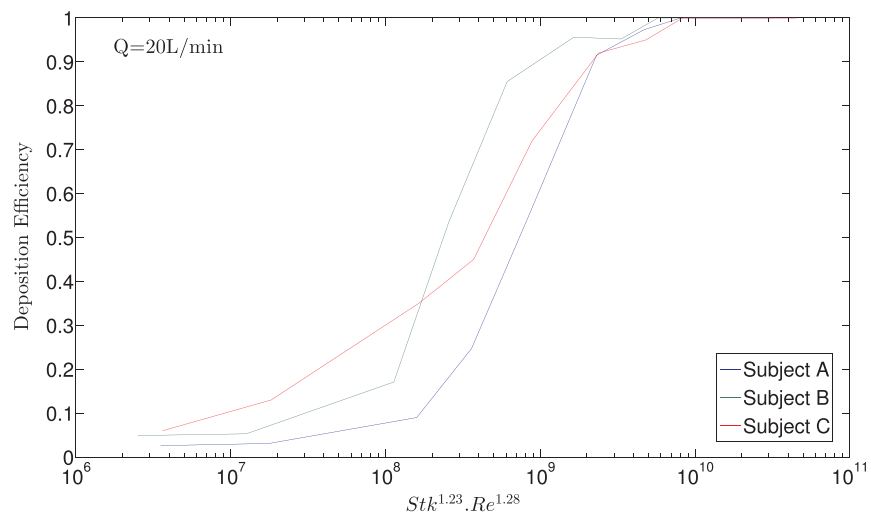

(c)

Fig. 10. Particle deposition efficiency plotted against non-dimensional fluid-particle dynamic parameter with characteristic diameter defined as $d_{c}=\sqrt{V / L}$, (L. Golshahi, M. Noga, et al., 2011).

suspended particles.

The Wall-shear stress gradient as an indicator of disturbed near-wall flow field has been used in the past (Hyun, Kleinstreuer, \& Archie, 2001) to indicate potential sites of particle deposition. Fig. 9 shows micro-particle deposition pattern and a map of the wall-shear stress gradient for Subject A. Some local prominent accumulations of deposited particles, also called hot spots, coincide with local maxima of the wall-shear stress gradient (see Fig. 9 a, b or c). For example where the mechanism of deposition is impaction (see b1 of Fig. 9b), the correlation with high wall-shear stress gradient is corroborated. In contrast, when secondary flows cause deposition the correlation is not observed (see a1 of Fig. 9a). 


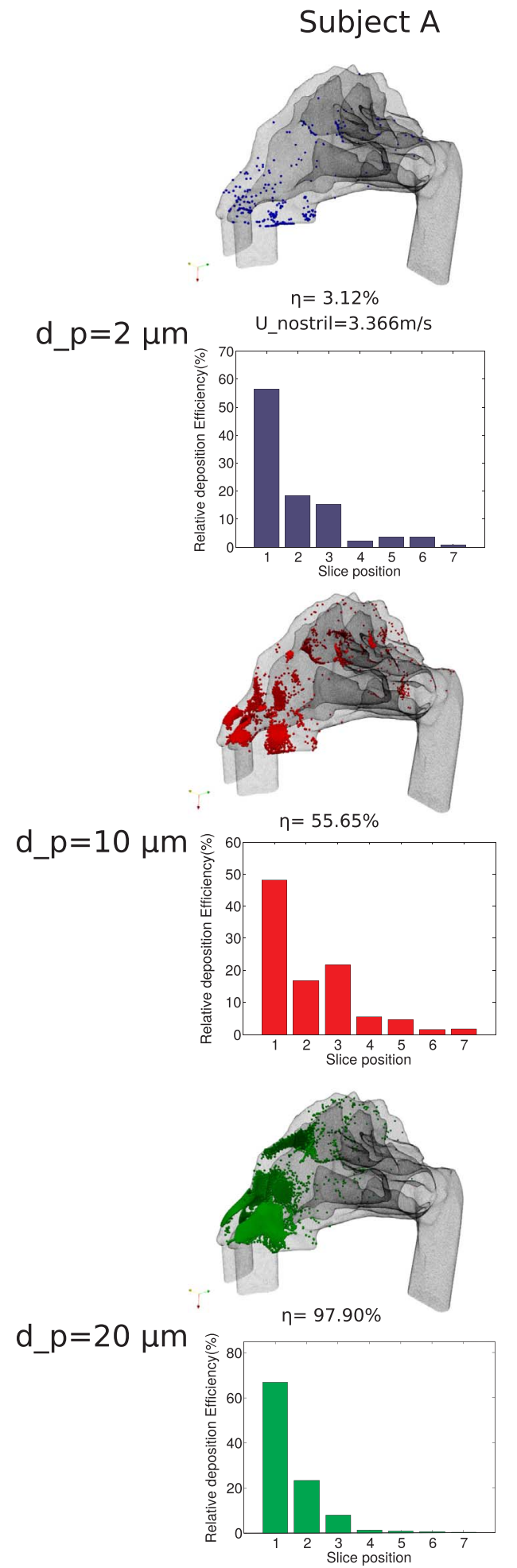

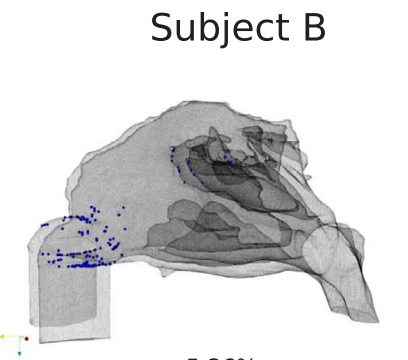

$\eta=5.36 \%$
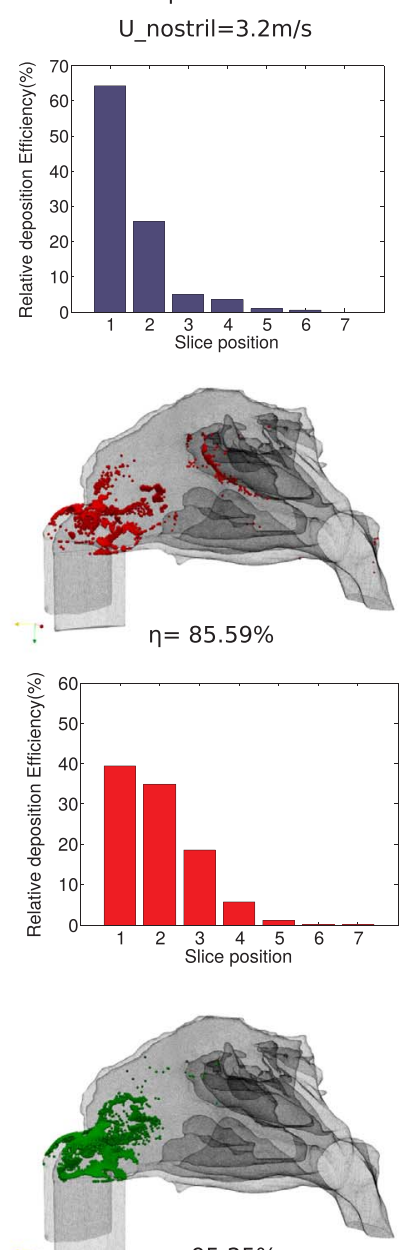

$\eta=95.25 \%$

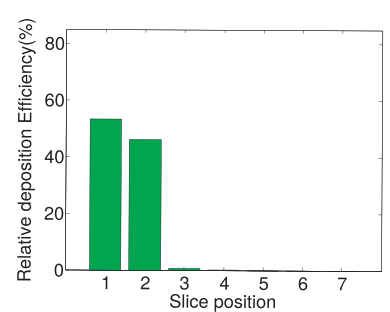

Subject C

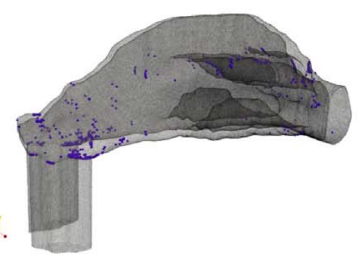

$\eta=9.95 \%$
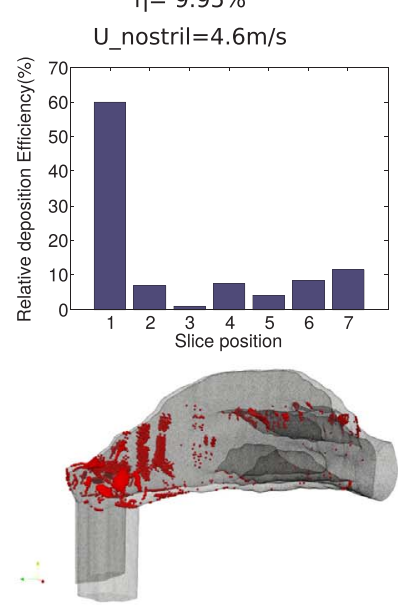

$\eta=85.36 \%$
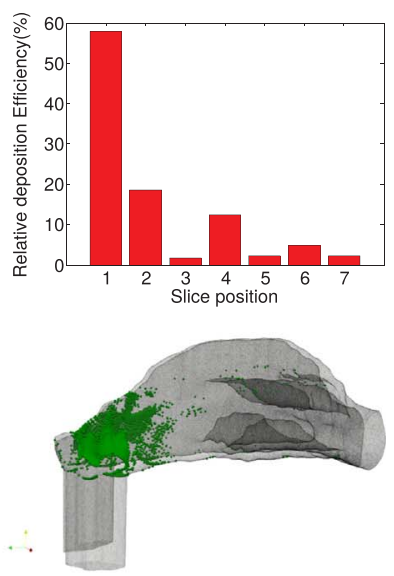

$\eta=98.57 \%$

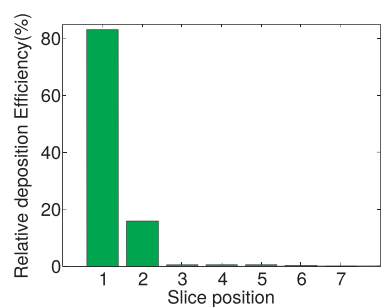

Fig. 11. Particle deposition pattern and particle deposition efficiency as a function of slice position (see Figs. 5-7) for the three subjects at a constant inlet flow rate of $20 \mathrm{~L} / \mathrm{min}$. 


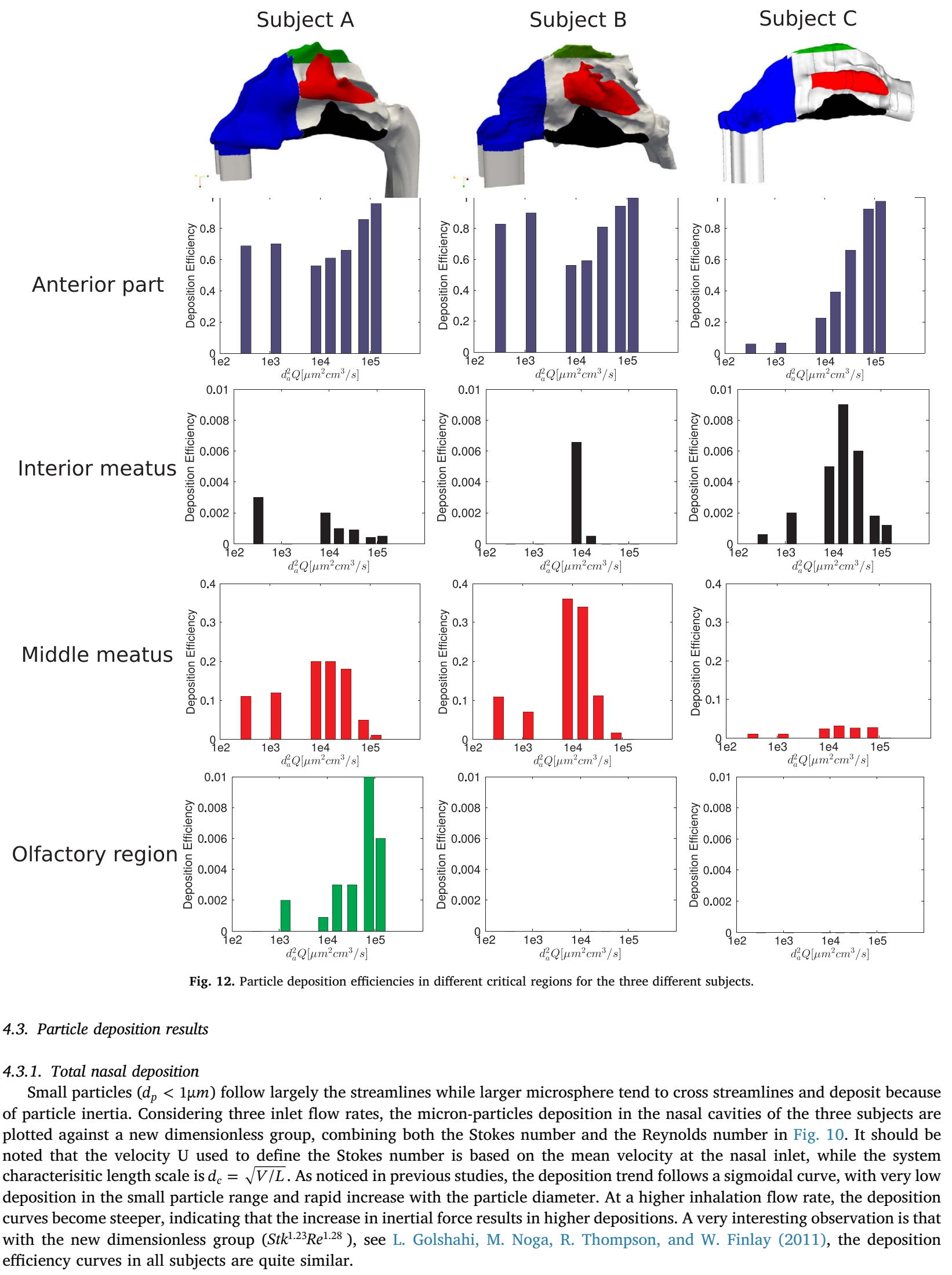




\subsubsection{Spatial deposition distribution}

The spatial deposition distributions of the particles in the nasal airways of the three subjects for different micron particle diameters are shown in Fig 11. The influence of the local airflow field on particle deposition is evident. Enhanced deposition in the anterior section of the nasal airway passage occurred in all three subjects. The deposition on the surface covering up to slice 2-2 is over $60 \%$ of the total deposition in all subjects. Apart from the anterior sections of the nasal airways covering mostly the nasal valve, concentrated deposition was also noticed in the middle meatus in all three subjects. In subject A, apart from these two sections, a small percentage of the particles deposited in the anterior section of the superior turbinates. The concentrated deposition site for particle size $10 \mu \mathrm{m}$ was in the upper turbinates while for particle size $20 \mu \mathrm{m}$ it moved to the anterior turbinates. This could be attributed to the bulk flow through the superior meatus in subject A (see Fig 5). As the particle size increased, the number of particles deposited in the nasal cavity increased to more than $50 \%$ for $10 \mu \mathrm{m}$ particles and to more than $90 \%$ for $20 \mu \mathrm{m}$ particles, due to inertial impaction.

\subsubsection{Regional deposition distribution}

The regional deposition efficiency was estimated for each section as a function of the inertial parameter $d_{a}^{2} Q$. The sectional deposition results are shown in Fig 12. As expected, maximum deposition occurred in the anterior section of the nasal airway for all three subjects (see blue colored surfaces in Fig 12). The deposition in the anterior surface is high for larger particles at lower inhalation flow rates. The deposition decreases for small particles with high inhalation flow rates and large particles with medium inhalation flow rates. The deposition in the inferior meatus is low in all subjects. The deposition in the olfactory region is very low for subject A and almost non-exististent in subject B and subject C. Clearly, for direct delivery to the brain via the olfactory region, directional inhalation of nano-particles would be required.

\section{Conclusions}

The human nose generally works as a fine filter of pollutants; but, it could also be used for the delivery of medicine to treat various diseases. One example would be targeting the olfactory region for deposited drug to cross the blood-brain-barrier to reach the central nervous system. Unfortunately the human nasal airways are complex and may differ greatly between subjects. Thus, considering a basic range of inhalation flow rates and microspheres, the airflow field, wall shear stress distribution and coupled particle transport/ deposition in three subject-specific nasal models have been simulated and analyzed in detail. Specifically, the airflow and particletrajectory equations were solved in parallel with the in-house, multi-purpose Alya program at the Barcelona Supercomputing Center.

The geometrically complex nasal airways generated a large variety of airflow fields, where among them the three subject models exhibit both similar as well as diverse flow structures and wall shear stress distributions, all related to the coupled particle transport and deposition. Nevertheless, with the new Stokes-Reynolds-number group, $S t k^{1.23} \operatorname{Re}^{1.28}$, the total deposition-efficiency curves for all three subjects and flow rates almost collapsed to a single function. However, local particle deposition efficiencies differed significantly for the three subjects and particle diameters $d_{p}=2,10$, and $20 \mu \mathrm{m}$.

Only one of the three subject-specific olfactory regions received, at relatively high values of the inertial parameter $d_{a}^{2} Q$, some inhaled microspheres. Thus, to achieve direct drug delivery to the brain via the olfactory region, future work will focus on the dynamics of inhaled nanoparticles using a modified inhaler device for targeting.

\section{Acknowledgments}

The authors acknowledge Dr. Rick Corley and colleagues at Pacific Northwest National Laboratory for providing the subject B nasal surface geometry and Dr. Edgar Matida and Dr. Matthew Johnson at Carleton University for providing the subject C nasal surface geometry.

\section{References}

Alya System, Large Scale Computational Mechanics, 2000. URL: 〈http://www.bsc.es/computer-applications/alya-system〉.

ANSYS Meshing User's Guide, 2000. 〈http://148.204.81.206/Ansys/150/ANSYSMeshingUsersGuide.pdf〉.

Asgharian, B., Price, O. T., Schroeter, J. D., Kimbell, J. S., \& Singal, M. (2012). A lung dosimetry model of vapor uptake and tissue disposition. Inhalation Toxicology, 24, $182-193$.

Bennett, W. D., \& Zeman, K. L. (2005). Effect of race on fine particle deposition for oral and nasal breathing. Inhalation Toxicology, 17, 641-648.

Calmet, H., Gambaruto, A. M., Bates, A. J., Vázquez, M., Houzeaux, G., \& Doorly, D. J. (2016). Large-scale cfd simulations of the transitional and turbulent regime for the large human airways during rapid inhalation. Computers in Biology and Medicine, 69, 166-180.

Cheng, K. H., Cheng, Y. S., Yeh, H. C., \& Swift, D. L. (1995). Deposition of ultrafine aerosols in the head airways during natural breathing and during simulated breath holding using replicate human upper airway casts. Aerosol Science and Technology, 23, 465-474.

Cheng, K.-H., Cheng, Y.-S., Yeh, H.-C., Guilmette, R. A., Simpson, S. Q., Yang, Y.-H., \& Swift, D. L. (1996a). In vivo measurements of nasal airway dimensions and ultrafine aerosol deposition in the human nasal and oral airways. Journal of Aerosol Science, 27, 785-801.

Cheng, Y. S. (2003). Aerosol deposition in the extrathoracic region. Aerosol Science \& Technology, 37, 659-671.

Cheng, Y. S., Holmes, T. D., Gao, J., Guilmette, R. A., Li, S., Surakitbanharn, Y., \& Rowlings, C. (2001). Characterization of nasal spray pumps and deposition pattern in a replica of the human nasal airway. Journal of Aerosol Medicine, 14, 267-280.

Cheng, Y. S., Yeh, H. C., Guilmette, R. A., Simpson, S. Q., Cheng, K. H., \& Swift, D. L. (1996b). Nasal deposition of ultrafine particles in human volunteers and its relationship to airway geometry. Aerosol Science and Technology, 25, 274-291.

Cheng, Y.-S., Yeh, H. C., \& Swift, D. L. (1991). Aerosol deposition in human nasal airway for particles 1 nm to $20 \mu m$ : A model study. Radiation Protection Dosimetry, 38 , 41-47.

Colomés, O., Badia, S., Codina, R., \& Principe, J. (2015). Assessment of variational multiscale models for the large eddy simulation of turbulent incompressible flows. 
Computer Methods in Applied Mechanics and Engineering, 285, 32-63.

Corey, J. P., Gungor, A., Liu, X., Nelson, R., \& Fredberg, J. (1998). Normative standards for nasal cross-sectional areas by race as measured by acoustic rhinometry. Otolaryngology-Head and Neck Surgery, 119, 389-393 (9781997).

Corey, J. P., Gungor, A., Nelson, R., Fredberg, J., \& Lai, V. (1997). A comparison of the nasal cross-sectional areas and volumes obtained with acoustic rhinometry and magnetic resonance imaging. Otolaryngology-Head and Neck Surgery, 117, 349-354.

Corley, R. A., Kabilan, S., Kuprat, A. P., Carson, J. P., Jacob, R. E., Minard, K. R., ... Einstein, D. R. (2015). Comparative risks of aldehyde constituents in cigarette smoke using transient computational fluid dynamics/physiologically based pharmacokinetic models of the rat and human respiratory tracts. Toxicological Sciences, $146,65-88$.

Dastan, A., Abouali, O., \& Ahmadi, G. (2014). Cfd simulation of total and regional fiber deposition in human nasal cavities. Journal of Aerosol Science, 69, 132-149.

Dhuria, S. V., Hanson, L. R., \& Frey, W. H. (2010). Intranasal delivery to the central nervous system: Mechanisms and experimental considerations. Journal of Pharmaceutical Sciences, 99, 1654-1673.

Doorly, D. J., Taylor, D. J., Gambaruto, A. M., Schroter, R. C., \& Tolley, N. (2008). Nasal architecture: Form and flow. Philosophical Transactions Royal Society A, 366, 3225-3246.

Field, D. A. (1988). Laplacian smoothing and delaunay triangulations. Communications in Applied Numerical Methods, 4, 709-712.

Folk, M., Cheng, A., Yates, K., (1999). Hdf5: A file format and i/o library for high performance computing applications. In Proceedings of Supercomputing, volume 99, pp. 5-33.

Gambaruto, A. M., Doorly, D. J., \& Yamaguchi, T. (2010). Wall shear stress and near-wall convective transport: Comparisons with vascular remodelling in a peripheral graft anastomosis. Journal of Computational Physics, 229, 5339-5356.

Ganser, G. (1993). A rational approach to drag prediction of spherical and nonspherical particles. Powder Technology, 77, $143-152$.

Garcia, G. J., Bailie, N., Martins, D. A., \& Kimbell, J. S. (2007). Atrophic rhinitis: A cfd study of air conditioning in the nasal cavity. Journal of Applied Physiology, 103, 1082-1092 (17569762).

Garcia, G. J., Schroeter, J. D., \& Kimbell, J. S. (2015). Olfactory deposition of inhaled nanoparticles in humans. Inhalation Toxicology, $27,394-403$.

Garcia, G. J., Schroeter, J. D., Segal, R. A., Stanek, J., Foureman, G. L., \& Kimbell, J. S. (2009). Dosimetry of nasal uptake of water-soluble and reactive gases: A first study of interhuman variability. Inhalation Toxicology, 21, 607-618.

Garcia, G. J., Tewksbury, E. W., Wong, B. A., \& Kimbell, J. S. (2009). Interindividual variability in nasal filtration as a function of nasal cavity geometry. Journal of Aerosol Medicine and Pulmonary Drug Delivery, 22, 139-156.

Ghalati, P. F., Keshavarzian, E., Abouali, O., Faramarzi, A., Tu, J., \& Shakibafard, A. (2012). Numerical analysis of micro-and nano-particle deposition in a realistic human upper airway. Computers in Biology and Medicine, 42, 39-49.

Golshahi, L., Noga, M., Thompson, R., \& Finlay, W. (2011). In vitro deposition measurement of inhaled micrometer-sized particles in extrathoracic airways of children and adolescents during nose breathing. Journal of Aerosol Science, 42, 474-488.

Golshahi, L., Noga, M. L., Thompson, R. B., \& Finlay, W. H. (2011). In vitro deposition measurement of inhaled micrometer-sized particles in extrathoracic airways of children and adolescents during nose breathing. Journal of Aerosol Science, 42, 474-488.

Guilmette, R. A., Cheng, Y. S., \& Griffith, W. C. (1997). Characterising the variability in adult human nasal airway dimensions. The Annals of Occupational Hygiene, 41, $491-496$.

Houzeaux, G., Aubry, R., \& Vázquez, M. (2011). Extension of fractional step techniques for incompressible flows: The preconditioned orthomin(1) for the pressure schur complement. Computers \& Fluids, 44, 297-313.

Houzeaux, G., de la Cruz, R., Owen, H., \& Vázquez, M. (2013). Parallel uniform mesh multiplication applied to a navier-stokes solver. Computers \& Fluids, 80, 142-151.

Houzeaux, G., Garcia-Gasulla, M., Cajas, J., Artigues, A., Olivares, E., Labarta, J., \& Vázquez, M. (2016). Dynamic load balance applied to particle transport in fluids. International Journal of Computational Fluid Dynamics.

Houzeaux, G., \& Principe, J. (2008). A variational subgrid scale model for transient incompressible flows. International Journal of Computational Fluid Dynamics, 22, $135-152$.

Houzeaux, G., Vázquez, M., (2008). Parallel implementation of a predictor-corrector scheme for the solution of the navier-stokes equations.

Hyun, S., Kleinstreuer, C., \& Archie, J., Jr. (2001). Computational particle-hemodynamics analysis and geometric reconstruction after carotid endarterectomy. Computers in Biology and Medicine, 31, 365-384.

Illum, L. (2000). Transport of drugs from the nasal cavity to the central nervous system. European Journal of Pharmaceutical Sciences, 11, 1-18.

Illum, L., Tsuda, A., \& Gehr, P. (2015). The potential for nose-to-brain delivery of drugs. Boca Raton, FL: CRC Press/Taylor and Francis Group.

Inthavong, K., Tian, Z. F., Li, H. F., Tu, J. Y., Yang, W., Xue, C. L., \& Li, C. G. (2006). A numerical study of spray particle deposition in a human nasal cavity. Aerosol Science and Technology, 40, 1034-1045.

Inthavong, K., Tian, Z. F., Tu, J. Y., Yang, W., \& Xue, C. (2008a). Optimising nasal spray parameters for efficient drug delivery using computational fluid dynamics. Computers in Biology and Medicine, 38, 713-726.

Inthavong, K., Wen, J., Tian, Z., \& Tu, J. (2008). Numerical study of fibre deposition in a human nasal cavity. Journal of Aerosol Science, 39, $253-265$.

Kabilan, S., Suffield, S. R., Recknagle, K. P., Jacob, R. E., Einstein, D. R., Kuprat, A. P., ... Hines, S. (2016). Computational fluid dynamics modeling of bacillus anthracis spore deposition in rabbit and human respiratory airways. Journal of Aerosol Science, 99, 64-77.

Karypis, G. Metis: Serial graph partitioning and fill-reducing matrix ordering, 1995-2015. Available at: URL 〈http://glaros.dtc.umn.edu/gkhome/views/metis〉.

Keeler, J. A., Patki, A., Woodard, C. R., \& Frank-Ito, D. O. (2016). A computational study of nasal spray deposition pattern in four ethnic groups. Journal of Aerosol Medicine and Pulmonary Drug Delivery, 29, 153-166.

Kelly, J. T., Asgharian, B., Kimbell, J. S., \& Wong, B. A. (2004). Particle deposition in human nasal airway replicas manufactured by different methods. part i: Inertial regime particles. Aerosol Science and Technology, 38, 1063-1071.

Kelly, J. T., Asgharian, B., Kimbell, J. S., \& Wong, B. A. (2004aa). Particle deposition in human nasal airway replicas manufactured by different methods. part i: Inertial regime particles. Aerosol Science and Technology, 38, 1063-1071.

Kelly, J. T., Asgharian, B., Kimbell, J. S., \& Wong, B. A. (2004bb). Particle deposition in human nasal airway replicas manufactured by different methods. Part ii: Ultrafine particles. Aerosol Science and Technology, 38, 1072-1079.

Kesavan, J., Bascom, R., Laube, B., \& Swift, D. L. (2000). The relationship between particle deposition in the anterior nasal passage and nasal passage characteristics. Journal of Aerosol Medicine, 13, 17-23.

Kesavanathan, J., Bascom, R., \& Swift, D. L. (1998). The effect of nasal passage characteristics on particle deposition. Journal of Aerosol Medicine, 11, 27-39.

Kesavanathan, J., \& Swift, D. L. (1998). Human nasal passage particle deposition: The effect of particle size, flow rate, and anatomical factors. Aerosol Science and Technology, 28, 457-463.

Keyhani, K., Scherer, P. W., \& Mozell, M. M. (1997). A numerical model of nasal odorant transport for the analysis of human olfaction. Journal of Theoretical Biology, $186,279-301$.

Kim, S. K., Na, Y., Kim, J.-I., \& Chung, S.-K. (2013). Patient specific cfd models of nasal airflow: Overview of methods and challenges. Journal of Biomechanics, 46, 299-306.

Kimbell, J. S., Frank, D. O., Laud, P., Garcia, G., \& Rhee, J. S. (2013). Changes in nasal airflow and heat transfer correlate with symptom improvement after surgery for nasal obstruction. Journal of Biomechanics, 46, 2634-2643.

Kimbell, J. S., Segal, R. A., Asgharian, B., Wong, B. A., Schroeter, J. D., Southall, J. P., ... Miller, F. J. (2007). Characterization of deposition from nasal spray devices using a computational fluid dynamics model of the human nasal passages. Journal of Aerosol Medicine, 20, 59-74.

Kleinstreuer, C., Feng, Y., \& Childress, E. (2014). Drug-targeting methodologies with applications: A review. World Journal of Clinical Cases, 2, $742-756$ (25516850).

Kolanjiyil, A. V., \& Kleinstreuer, C. (2013). Nanoparticle mass transfer from lung airways to systemic regions-part i: Whole-lung aerosol dynamics. Journal of Biomechanical Engineering, 135, 121003.

Kolanjiyil, A. V., \& Kleinstreuer, C. (2016). Computationally efficient analysis of particle transport and deposition in a human whole-lung-airway model. part i: Theory and model validation. Computers in Biology and Medicine, 79, 193-204. 
Kolanjiyil, A. V., Kleinstreuer, C., \& Sadikot, R. T. (2016). Computationally efficient analysis of particle transport and deposition in a human whole-lung-airway model. part ii: Dry powder inhaler application. Computers in Biology and Medicine, 84, 247-253.

Leong, S. C., Chen, X. B., Lee, H. P., \& Wang, D. Y. (2010). A review of the implications of computational fluid dynamic studies on nasal airflow and physiology. Rhinology, 48, 139 (20502749).

Liu, Y., Johnson, M. R., Matida, E. A., Kherani, S., \& Marsan, J. (2009). Creation of a standardized geometry of the human nasal cavity. Journal of Applied Physiology, 106, 784-795.

Liu, Y., Matida, E. A., \& Johnson, M. R. (2010). Experimental measurements and computational modeling of aerosol deposition in the carleton-civic standardized human nasal cavity. Journal of Aerosol Science, 41, 569-586.

Lohner, R., Mut, F., Cebral, J., Aubry, R., \& Houzeaux, G. (2011). Deflated preconditioned conjugate gradient solvers for the pressure-poisson equation: Extensions and improvements. International Journal of Numerical Methods in Engineering, 87, 2-14.

Menache, M. G., Hanna, L. M., Gross, E. A., Lou, S.-R., Zinreich, S. J., Leopold, D. A., ... Miller, F. J. (1997). Upper respiratory tract surface areas and volumes of laboratory animals and humans: Considerations for dosimetry models. Journal of Toxicology and Environmental Health, 50, $475-506$.

Mistry, A., Stolnik, S., \& Illum, L. (2009). Nanoparticles for direct nose-to-brain delivery of drugs. International Journal of Pharmaceutics, 379, $146-157$.

Morris, J. B., HAssETT, D. N., \& Blanchard, K. T. (1993). A physiologically based pharmacokinetic model for nasal uptake and metabolism of nonreactive vapors. Toxicology and Applied Pharmacology, 123, 120-129.

Olivares, E., Houzeaux, G., (2016). Time integration schemes comparation for particles transport. In ECCOMAS16, Crete (Greece).

Piomelli, U., \& Balaras, E. (2002). Wall-layer models for large-eddy simulations. Annual Review of Fluid Mechanics, 34, $349-374$.

Rasmussen, T. R., Andersen, A., \& Pedersen, O. F. (2000). Particle deposition in the nose related to nasal cavity geometry. Rhinology, 38, 102-107 (11072654).

Rhee, J. S., Pawar, S. S., Garcia, G. J., \& Kimbell, J. S. (2011). Toward personalized nasal surgery using computational fluid dynamics. Archives of Facial Plastic Surgery, 13, 305-310.

Robinson, S. K. (1991). Coherent motions in the turbulent boundary layer. Annual Review of Fluid Mechanics, 23, 601-639.

Schroeter, J. D., Garcia, G. J., \& Kimbell, J. S. (2010). A computational fluid dynamics approach to assess interhuman variability in hydrogen sulfide nasal dosimetry. Inhalation Toxicology, 22, 277-286.

Schroeter, J. D., Garcia, G. J., \& Kimbell, J. S. (2011). Effects of surface smoothness on inertial particle deposition in human nasal models. Journal of Aerosol Science, 42 , 52-63.

Schroeter, J. D., Kimbell, J. S., \& Asgharian, B. (2006). Analysis of particle deposition in the turbinate and olfactory regions using a human nasal computational fluid dynamics model. Journal of Aerosol Medicine, 19, 301-313.

Schroeter, J. D., Tewksbury, E. W., Wong, B. A., \& Kimbell, J. S. (2015a). Experimental measurements and computational predictions of regional particle deposition in a sectional nasal model. Journal of Aerosol Medicine and Pulmonary Drug Delivery, 28, 20-29.

Schroeter, J. D., Tewksbury, E. W., Wong, B. A., \& Kimbell, J. S. (2015b). Experimental measurements and computational predictions of regional particle deposition in a sectional nasal model. Journal of Aerosol Medicine and Pulmonary Drug Delivery, 28, 20-29.

Schroete, J. D., Kimbell, J. S., Gross, E. A., Willso, G. A., Dorman, D. C., Tan, Y.-M., \& Clewell, H. J., III (2008). Application of physiological computational fluid dynamics models to predict interspecies nasal dosimetry of inhaled acrolein. Inhalation Toxicology, 20, $227-243$.

Segal, R. A., Kepler, G. M., \& Kimbell, J. S. (2008). Effects of differences in nasal anatomy on airflow distribution: A comparison of four individuals at rest. Annals of Biomedical Engineering, 36, 1870-1882.

Shanley, K. T., Zamankhan, P., Ahmadi, G., Hopke, P. K., \& Cheng, Y.-S. (2008). Numerical simulations investigating the regional and overall deposition efficiency of the human nasal cavity. Inhalation Toxicology, 20, 1093-1100.

Shi, H. (2007). Numerical simulation of airflow, particle deposition and drug delivery in a representative human nasal airway model (Ph.D. Dissertation). Raleigh, NC, USA: NC State University.

Shi, H., Kleinstreuer, C., \& Zhang, Z. (2007a). Modeling of inertial particle transport and deposition in human nasal cavities with wall roughness. Journal of Aerosol Science, 38, 398-419.

Shi, H., Kleinstreuer, C., \& Zhang, Z. (2007b). Modeling of inertial particle transport and deposition in human nasal cavities with wall roughness. Journal of Aerosol Science, 38, 398-419.

Shi, H., Kleinstreuer, C., \& Zhang, Z. (2008a). Dilute suspension flow with nanoparticle deposition in a representative nasal airway model. Physics of Fluids, 20, 013301.

Shi, H., Kleinstreuer, C., \& Zhang, Z. (2008b). Dilute suspension flow with nanoparticle deposition in a representative nasal airway model. Physics of Fluids, 20, 013301 (1994-present).

Soto, O., Löhner, R., \& Camelli, F. (2003). A linelet preconditioner for incompressible flow solvers. International Journal of Numerical Methods for Heat \& Fluid Flow, 13, 133-147.

Storey-Bishoff, J., Noga, M., \& Finlay, W. (2008). Deposition of micrometer-sized aerosol particles in infant nasal airway replicas. Journal of Aerosol Science, 39, 1055-1065.

Thorne, R. G., Emory, C. R., Ala, T. A., \& Frey, W. H. (1995). Quantitative analysis of the olfactory pathway for drug delivery to the brain. Brain Research, 692, $278-282$.

Thorne, R. G., Pronk, G. J., Padmanabhan, V., \& Frey, W., 2nd (2004). Delivery of insulin-like growth factor-i to the rat brain and spinal cord along olfactory and trigeminal pathways following intranasal administration. Neuroscience, 127, 481-496.

Wang, Z., Hopke, P. K., Ahmadi, G., Cheng, Y.-S., \& Baron, P. A. (2008). Fibrous particle deposition in human nasal passage: The influence of particle length, flow rate, and geometry of nasal airway. Journal of Aerosol Science, 39, 1040-1054.

Wiesmiller, K., Keck, T., Leiacker, R., Sikora, T., Rettinger, G., \& Lindemann, J. (2003). The impact of expiration on particle deposition within the nasal cavity. Clinical Otolaryngology \& Allied Sciences, 28, 304-307.

Xi, J., Kim, J., Si, X. A., Corley, R. A., \& Zhou, Y. (2016). Modeling of inertial deposition in scaled models of rat and human nasal airways: Towards in vitro regional dosimetry in small animals. Journal of Aerosol Science, 99, 78-93.

Xi, J., \& Longest, P. (2008). Numerical predictions of submicrometer aerosol deposition in the nasal cavity using a novel drift flux approach. International Journal of Heat and Mass Transfer, 51, 5562-5577.

Zhang, Z., \& Kleinstreuer, C. (2011). Computational analysis of airflow and nanoparticle deposition in a combined nasaloraltracheobronchial airway model. Journal of Aerosol Science, 42, 174-194.

Zwartz, G. J., \& Guilmette, R. A. (2001). Effect of flow rate on particle deposition in a replica of a human nasal airway. Inhalation Toxicology, 13, 109-127. 